\title{
A Tool for Automated Design and Evaluation of Habitat Interior Layouts
}

\author{
Matthew A. Simon \\ NASA Langley Research Center, Hampton, VA, 23681, USA \\ Dr. Alan W. Wilhite \\ Georgia Institute of Technology, Hampton, VA, 23666, USA
}

\begin{abstract}
The objective of space habitat design is to minimize mass and system size while providing adequate space for all necessary equipment and a functional layout that supports crew health and productivity. Unfortunately, development and evaluation of interior layouts is often ignored during conceptual design because of the subjectivity and long times required using current evaluation methods (e.g., human-in-the-loop mockup tests and in-depth CAD evaluations). Early, more objective assessment could prevent expensive design changes that may increase vehicle mass and compromise functionality. This paper describes a new interior design evaluation method to enable early, structured consideration of habitat interior layouts. This interior layout evaluation method features a comprehensive list of quantifiable habitat layout evaluation criteria, automatic methods to measure these criteria from a geometry model, and application of systems engineering tools and numerical methods to construct a multi-objective value function measuring the overall habitat layout performance. In addition to a detailed description of this method, a C++/OpenGL software tool which has been developed to implement this method is also discussed. This tool leverages geometry modeling coupled with collision detection techniques to identify favorable layouts subject to multiple constraints and objectives (e.g., minimize mass, maximize contiguous habitable volume, maximize task performance, and minimize crew safety risks). Finally, a few habitat layout evaluation examples are described to demonstrate the effectiveness of this method and tool to influence habitat design.
\end{abstract}

\section{Nomenclature}

$\begin{array}{ll}\frac{\text { Acronyms }}{\text { ECLSS }=} & \begin{array}{l}\text { Environmental Control and Life } \\ \text { Support System }\end{array} \\ \text { EVA }= & \text { Extra-Vehicular Activity } \\ \text { ISA-GJK }= & \text { Incremental Separating Axis - Gilbert- } \\ & \text { Johnson-Keerthi } \\ \text { ISS } & \text { International Space Station }\end{array}$

$$
\begin{array}{ll}
\text { LSS } & =\text { Lunar Surface System } \\
\text { MPLM } & =\text { Multi-Purpose Logistics Module } \\
\text { PCM } & =\text { Pressurized Core Module } \\
\text { PSO } & =\text { Particle Swarm Optimizer }
\end{array}
$$

\section{Introduction}

$\mathrm{D}^{\mathrm{s}}$ esigning habitats for crewed in-space or planetary surface missions is a complex, highly constrained task with many conflicting objectives. Habitats must provide a living volume appropriate for the mission duration and all of the functions and consumables required to support crew (e.g., a breathable atmosphere, clean water, food, a place to sleep, workstations to support crew tasks, etc.) Designers must ensure all of these systems are included and properly integrated while minimizing mass and cost. This is critical as habitats are often large, massive elements which must be pushed through most of a mission's propulsive maneuvers to support the crew. This large 'gear ratio' drives the design of launch vehicles and propulsion stages and often drives the overall cost and complexity of a mission. Therefore, the optimization of habitat designs is an important aspect of the development of any human space exploration mission.

The habitat design process involves the selection, sizing, and arrangement of the interior equipment and logistics required for a mission into an interior layout, which must fit within an appropriately sized pressure shell. The objective of habitat designers is to minimize mass and vehicle size while providing adequate space and a functional layout for crew health and productivity ${ }^{1,2,3}$. The interior arrangement step of this process seeks to ensure that the interior equipment required for a crewed mission can be physically accommodated while providing the best 
compromise between performance metrics such as mass, volume, workflow efficiency, and habitability. Balancing interior layout performance is especially critical at the conceptual design so that feasibility issues, safety concerns, and requirements violations can be identified before they result in expensive design changes, increased mass growth, or reduced functionality. However, ensuring that an interior layout is effectively balanced is non-trivial as there is currently no comprehensive and timely method to measure the effectiveness of an interior layout and track the complex, conflicting habitat design objectives during conceptual design. This missing evaluation capability increases the uncertainty surrounding conceptual habitat designs and prevents further efforts to optimize habitat designs for improved exploration mission performance. These concerns led to current research to improve the interior layout evaluation process for conceptual design in pursuit of an end-to-end habitat optimization capability.

This paper outlines a new, structured method to quickly measure the effectiveness of habitat interior designs, allowing for comparison of layouts at conceptual design and enabling the previously undemonstrated capability to automate the generation of habitat interiors. This will increase the understanding of habitat interior concerns at conceptual design and potentially result in cost and performance improvements enabling long duration missions. Section II will describe the proposed interior layout evaluation process which was developed through application of a systems engineering methodology. The section will also describe the selection of evaluation criteria and how these feed together into a multi-criteria objective function measuring overall layout effectiveness. Section III describes how these criteria are quantified using collision detection algorithms, functional separation/collocation matrices, and the use of discrete grid-based iterative methods. Section IV discusses the structure of the computer program created to implement this method. Section V provides a description and results from the example problem used to demonstrate the method, and Section VI describes the remaining work to complete this research.

\section{Evaluation Method}

Current methods for evaluating the goodness of these layouts involve human-in-the-loop mockup tests, in-depth CAD evaluations, and subjective design evaluation studies ${ }^{1}$. However, none of these methods are currently compatible with the conceptual phase of design or automation because of the significant time required to prepare and evaluate each layout. These methods were compared to the NASA Systems Engineering Trade Study Process ${ }^{4}$ to identify gaps which must be addressed to improve the habitat interior layout evaluation process. The major improvements identified to enable fast habitat interior layout evaluation are:

- A comprehensive, automatically quantifiable set of evaluation criteria

- A mathematical, computer representation of layout geometry and subsystem characteristics

- A structured method to capture designer preferences

- A multi-criteria objective function providing an aggregate measure of overall layout effectiveness

In order to evaluate multiple layout alternatives quickly and repeatably, a structured systems engineeringderived process built around quantifying a multi-criteria objective function for alternative layouts has been developed. This process needed to implement each of these improvements and employ several time-saving techniques to achieve the desired evaluation time of seconds per layout. A two part process is proposed: Layout Generation and Layout Evaluation. First, a structured Layout Generation method is created to translate mission requirements into the required layout geometric 'building blocks' and then generate mathematically representable layouts alternatives from these pieces compatible with the evaluation process. Then each of the layout evaluation improvements can be integrated into a systems engineering-derived Layout Evaluation process to calculate an objective function value which measures the overall acceptability of each layout. Figure 1 and Figure 2 present the proposed combined process for layout generation and evaluation based upon the basic systems engineering trade study process described in Ref. 4, but customized to implement the desired improvements.

For illustration, the NASA Scenario 12.0 Pressurized Core Module (PCM) habitat from the NASA Constellation Lunar Surface Scenario (LSS) team study ${ }^{5}$ shown in Figure 1 is used to demonstrate each step. This habitat was designed as part of a three module lunar surface habitat to support four crewmembers for durations up to 180 days. The diverse mix of functions represented in the design will facilitate discussion of the chosen evaluation criteria and measurement methods.

\section{A. Layout Generation Process}

Figure 1 shows the Layout Generation process, which translates requirements into geometric representations of physical hardware that can then be constructed into layouts for evaluation. First, the design problem and any design constraints to be enforced are defined. Examples of design problem descriptions with constraints are: "redesign 


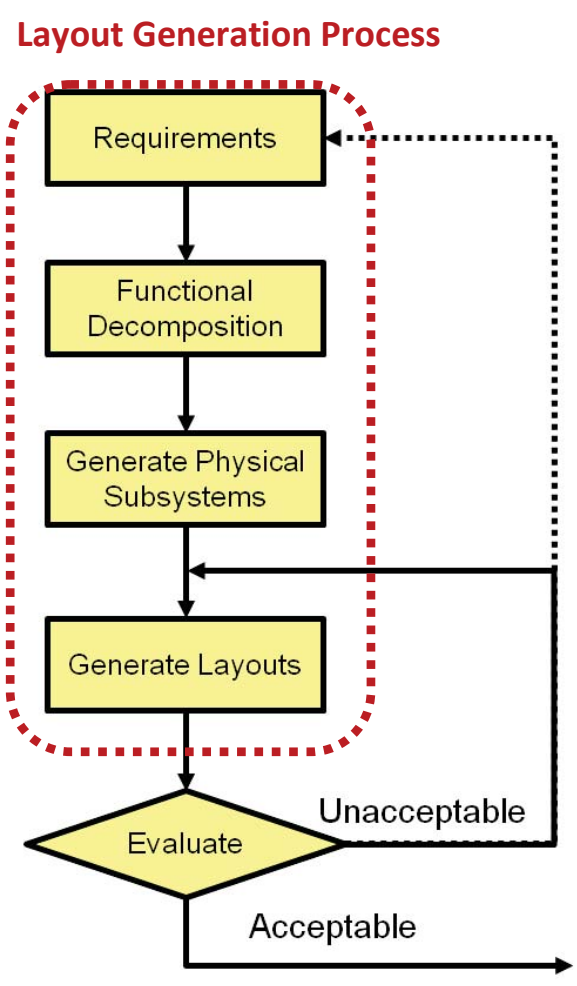

\section{EXAMPLE: \\ Scenario 12.0 Pressurized Core Module (PCM) ${ }^{5}$}

\section{Requirements:}

Improve upon NASA Scenario 12.0 PCM (lunar surface habitat for 180 days crew stays) design by rearranging layout

\section{Functional Decomposition:}

Galley, Wardroom, ECLSS, Stowage, Exercise, Waste and Hygiene, Medical, and Bio/Life Workstation

Generate Physical Subsystems:

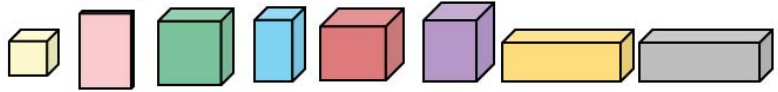

Generate Layouts:

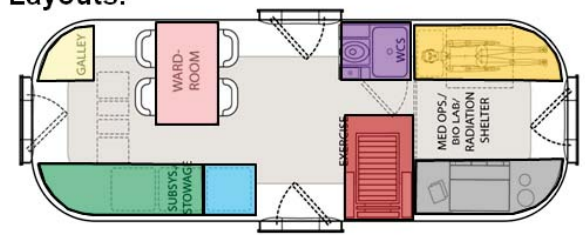

Evaluate:

Calculate Objective Function Value

- Evaluation Criteria (e.g. habitable volume ...)

- Designer Preferences (relative importance of evaluation criteria, desired values of criteria)

- Constraints (habitable volume, aisle width, ...)

Figure 1: Systems Engineering Basic Habitat Interior Layout Process

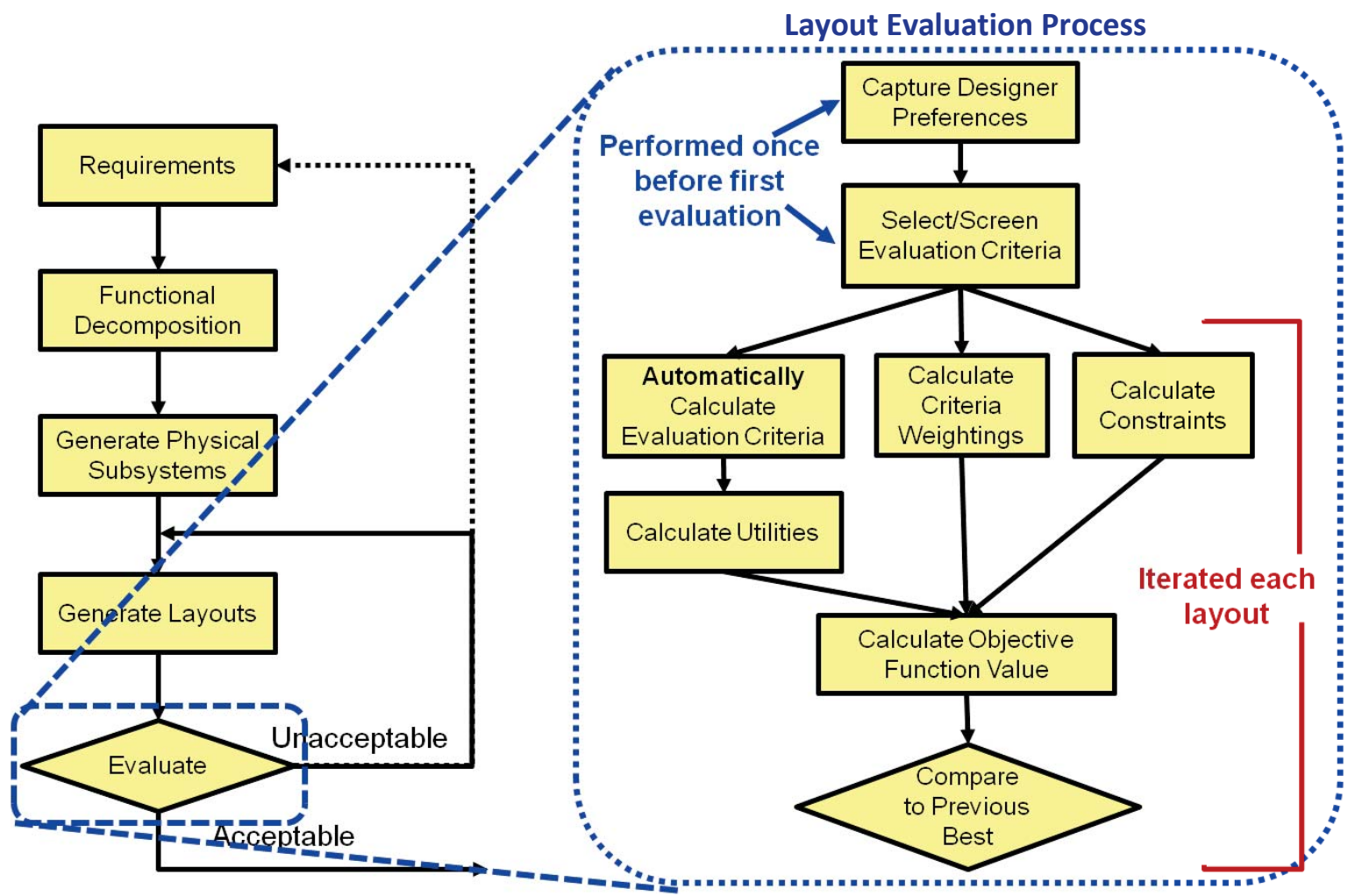

Figure 2: Proposed Systems Engineering Interior Layout Evaluation Process 
the interior of an existing International Space Station module keeping pressure vessel geometry constant" or "design a minimum mass lunar surface habitat." Additionally, specific mission requirements such as crewed duration, number of crew, and the destination must be defined. These requirements are necessary to dictate the required functions and set the appropriate context for the definition of designer preferences. In the case of the Scenario 12.0 PCM, the design problem statement and mission requirements are to redesign the interior of one module of a habitat supporting four crewmembers for 180 days of exploration of the lunar surface while maintaining the current pressure vessel and complement of subsystems.

The functions necessary to achieve the mission can be readily identified through a functional decomposition of the duration, mission tasks, and destination requirements established in the first step ${ }^{6,7}$. Fundamentally, most habitats provide the same functions, but the level of performance required within each function varies at certain breakpoints as mission requirements become more challenging. Comparing the mission requirements to these breakpoints leads to a direct determination of the hardware necessary for the required performance. This hardware is then distributed amongst the habitat modules (if multiple modules are assumed) based upon the specialized purpose of each habitat module. For example, in the Scenario 12.0 habitat concept, the 180 day duration at a lunar surface destination dictated the selection of appropriate subsystem hardware. Hardware items allocated to the PCM are focused on group and work activities and include: Galley, Wardroom, Life Support (partially closed), Stowage, Waste and Hygiene, Medical, and Biology/Life Science Research Station.

The 'generate physical subsystems' step involves the creation of a mathematical representation of hardware geometry and other characteristics of the equipment that influence its layout placement (e.g., tasks performed at hardware, mass, etc.) The historical habitat interior design process uses detailed CAD models or drafted drawings to represent the space taken up by the hardware in the layouts. These models can be powerful tools at the detail design phase, but their long creation times and complexity is often incompatible with fast layout evaluation. The proposed process uses a simple polyhedral representation of the hardware geometry derived from computer animation and video game programming which is well structured for use in layout creation and evaluation criteria calculation methods. By representing geometries as simple polyhedral objects specified by matrices of vertices and faces as shown in Figure 3, the overlap of geometries can be detected with standard collision detection algorithms to prevent the creation of unrealizable layouts ${ }^{8}$. Additionally, this polyhedral object representation allows for generation of layouts by simply manipulating the location and orientation of each subsystem through definition of translation and rotation matrices. What results is a fast, simple, mathematically operable method of constructing layout alternatives with relatively simple sets of data. Generating layouts in the following step simply involves assigning positions and orientations to each polyhedral object.

Additionally, using object-oriented programming allows for the embedding of detailed function and interface information together with the geometry data in arrays or matrices within an indexed object. The types of object information required include: the mass of an object, the function it belongs to, any separation or collocation relationships associated with the provided function, geometry and location of anthropometric envelopes reserved for human interaction with the object, and keep out zones for moving parts. Collocated storage of this information with geometry data facilitates straightforward calculation of evaluation criteria which track these characteristics in combination with geometry. The importance of this layout and data representation method will be discussed more in Section III. The full list of inputs provided to the evaluation process is shown in Table 1.

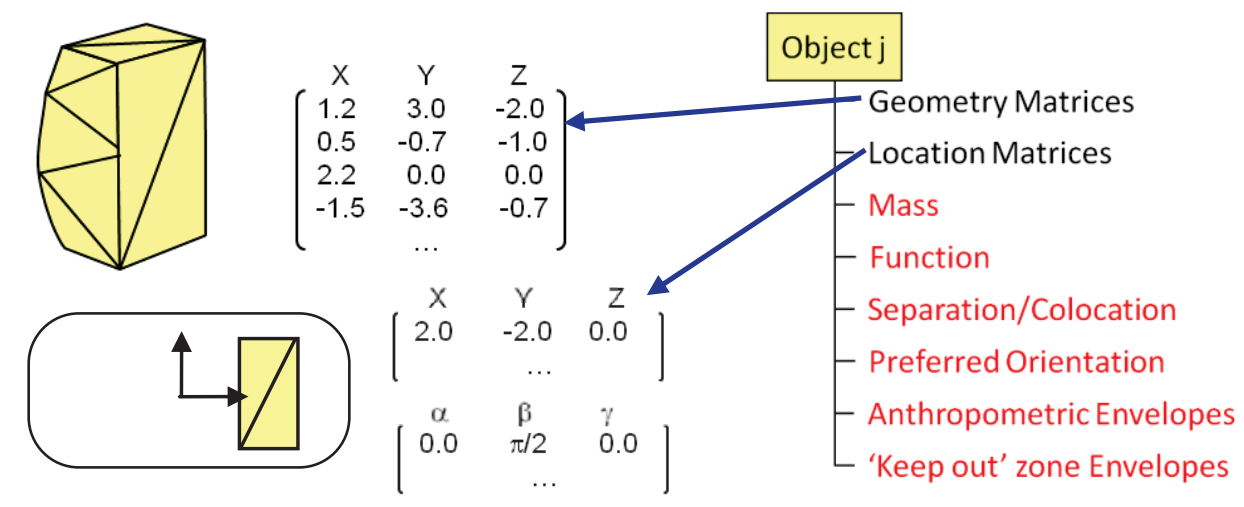

Figure 3: Mathematical Representation of Object Geometry Collocated with Detailed Information 
Table 1: Full List of Inputs Provided to the Evaluation Process

\begin{tabular}{|c|c|c|}
\hline $\begin{array}{l}\text { Mission Specifications } \\
\text { Destination (LEO, Moon, Mars, etc) } \\
\text { Duration of stay } \\
\text { Number of crew } \\
\text { Crew composition (scheduling) } \\
\text { EVA requirements } \\
\text { Landing requirements } \\
\text { Gravity orientation / magnitude } \\
\text { Surface dust requirements }\end{array}$ & $\begin{array}{l}\text { Pressure Vessel Geometry and Specifications } \\
\text { Basic dimensions } \\
\text { Shape } \\
\text { Orientation } \\
\text { Mass (input option available) } \\
\text { Floor height or floor area } \\
\text { Ceiling height } \\
\text { Number of hatches } \\
\text { Hatch locations } \\
\text { Hatch dimensions } \\
\text { Hatch type (EVA, inter-element, etc) } \\
\text { Diameter of endcap flattening for hatch } \\
\text { placement }\end{array}$ & $\begin{array}{l}\text { Component Geometry and Specifications } \\
\text { Dimensions } \\
\text { Vertices coordinates } \\
\text { Location (XYZ coordinates) } \\
\text { Orientation } \\
\text { Face data (which vertices in what } \\
\text { face, normal vectors) } \\
\text { Specification of front face(s) } \\
\text { Component Mass } \\
\text { Reserved volumes type } \\
\text { Reserved volume geometries } \\
\text { (vertices/lines/faces) } \\
\text { Function / task supported } \\
\text { Line runs required } \\
\text { Zoning requirements (privacy, noise, } \\
\text { clean/dirty) } \\
\text { Criticality of component } \\
\text { Frequency/duration of use }\end{array}$ \\
\hline
\end{tabular}

\section{B. Layout Evaluation Process}

Figure 2 shows the proposed Layout Evaluation process, which combines quantitative evaluation criteria measurements, subjective designer preferences, and design problem constraints into a single aggregate measure of the overall performance of a layout. In order to enable the comparison of multiple layouts at conceptual design leading to the automated generation of acceptable layouts, this process and its component steps must be carefully structured to reduce the evaluation time of each layout. First, before any layouts are evaluated, the three types of information are collected from the designer: 1) the relative importance of the evaluation criteria to establish criteria weightings, 2) desired values and acceptable ranges for each of the criteria to establish utility functions, and 3) any constraints the designer wishes to place on the design problem. By collecting this information before layout evaluation, it is possible to apply the same preferences and constraints to multiple layout concepts enabling applesto-apples comparisons. Each of these three is described in more detail.

\section{Criteria Weightings}

The relative importance of the evaluation criteria gives the designer an opportunity to customize the objective function to his/her preferences and the appropriate context of the design problem. This relative importance can be determined through expert elicitation using Analytic Hierarchy Process, a pair-wise comparison method which converts relative preferences scores between criteria to calculate normalized 'criteria weightings' for each criterion $^{9,10}$. These weightings are used to screen the evaluation criteria to those with the most effect on the objective function.

\section{Utility Functions}

In order to ensure each of the evaluation criteria values are combined in the objective function using common units (preventing criteria with high numerical values from dominating the solution), a function is used to normalize each calculated criteria value to a number between 0 and 1 (where 0 is lowest permissible performance and 1 is peak performance). This normalized score represents the designer's perceived 'utility' of a criteria value over the possible range of values. In most cases, linear utility functions are acceptable. However, linear improvement in the value of an evaluation criterion does not always correspond to a linear improvement in the designer's preference of that value. For example, at low values of habitable volume (e.g., $5 \mathrm{~m}^{3} /$ person) even slight volume increases may provide significantly improved human comfort, safety, or productivity. However, at high values (e.g., $40 \mathrm{~m}^{3} /$ person), even large increases in volume have diminishing returns as the volume becomes spacious to the point of being wasted. This diminishing return is reflected in the shape of the habitable utility function shown in Figure 4, which shows a negligible utility improvement past the value corresponding to the optimal amount of volume ${ }^{11}$. The method selected for collecting and applying this designer preference is Mid-Preference Level Splitting ${ }^{10}$ which uses a structured set of questions to shape the 'utility function' used to map evaluation criteria values to utilities. 


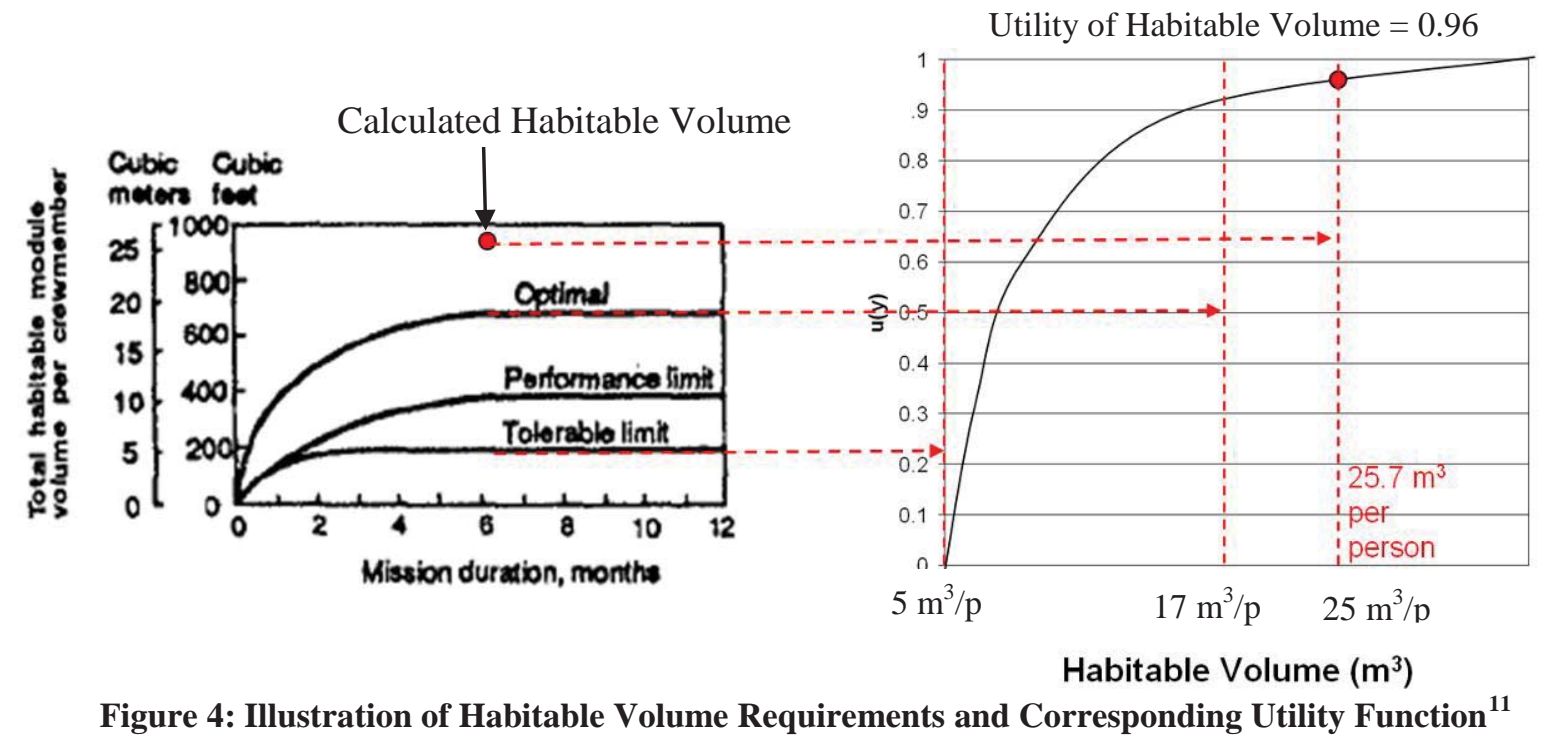

$\underline{\text { Constraints }}$

Constraints are limits specified by designer preference, physical realizability or human/spaceflight standards which are placed upon either the physical location of interior equipment or the values of the evaluation criteria measurements. Examples of constraints include hardcoded placement of certain pieces of hardware, prevention of overlapping hardware geometries, or human performance standard constraints like minimum volumes and minimum translation path widths. One of the difficulties in enforcing constraints on the interior layout is the decision of how to implement the constraints into the decision process. For hardware-hardware interferences, the simplest option would be to declare a configuration unfeasible if interferences occur. However, this will make automating the placement of interior components very difficult (e.g. if a layout is close to the optimum performance but a small clearance problem caused it to be missed). Selectively allowing interferences to some degree can improve traversal of the layout design space towards optimum configurations.

Penalty functions applied at the objective function level are the anticipated best method to implement constraints. Penalty functions return exponentially increasing large values as constraints are violated, which can be subtracted from the unconstrained weighted sum of evaluation criteria to prevent layout designs which violate constraints from being acceptable. Interior penalty functions increase before the constraint is violated to enforce 'hard' constraints which must be met for feasible designs. Exterior penalty functions are used for 'soft' constraints where slight violations are acceptable. In general, exterior penalty functions are anticipated to provide more flexibility in finding solutions. Additionally, all constraints can be relaxed during early iterations to prevent a lack of freedom for the optimization method to explore the design space and some schedule of the rate of increase of the penalty functions can be implemented to avoid local optima. For more information on external penalty functions see Ref. 12.

\section{Evaluation Criteria}

Apart from designer preferences, the only part of Figure 2 feeding into the objective function not defined yet is the automatically quantifiable evaluation criteria values. Investigation of previous evaluation methods identified that no comprehensive, expert approved list of quantifiable evaluation criteria capturing both engineering and human habitability concerns has been created ${ }^{6,13-16}$. In particular, the desire to improve consideration of habitability issues into the layout evaluation is well documented ${ }^{5,16-22}$. The development of a comprehensive set of automatically quantifiable evaluation criteria calculable from layout geometry and hardware functional characteristics is critical to ensuring high-quality habitat designs and speeding up the evaluation process.

The proposed evaluation criteria set is derived from existing quantitative and qualitative habitat and terrestrial architecture evaluation methods and human integration handbooks $1,6,14,15,19,23-26$. In order to ensure a comprehensive set of criteria was chosen, a structured screening process was used to assess whether a criterion was essential to the assessment of interior layouts. First, a comprehensive list of all possible habitat interior layout evaluation criteria was created from an extensive literature review of space habitat design ${ }^{7,11,27}$, space vehicle habitability ${ }^{6,14,24,26}$, industrial engineering, and terrestrial architecture references (particularly from Architectural Programming ${ }^{28}$ and a 
field of study focusing on automation in architecture, and Space Layout Planning ${ }^{29,30,31}$ ). Additionally, several of the more complete evaluation criteria sets used in existing habitat design literature were included ${ }^{6,13,14,15,24,32-34}$. The fully comprehensive list of possible criteria was then screened based upon the following list of desired characteristics. Criteria should be:

- As independent as possible from other criteria to prevent an over-emphasis on any particular measure ${ }^{10}$

- Explicitly dependent upon layout or pressure vessel geometry, which allows for the exclusion of many aesthetic criteria such as color or textures which can be changed with little to no impact after optimizing interior layouts

- $\quad$ Intuitive and easy to justify ${ }^{10}$

- Consistent with existing requirements ${ }^{11,35}$

Finally, as the habitat design experts are the target users of this methodology, the criteria should be habitat expert recognized and approved. This final step was achieved by vetting the resulting list of criteria with the NASA Johnson Space Center habitation and layout evaluation experts.

The resulting comprehensive evaluation criteria set is shown in Figure 5 grouped into the following four categories: Mass, Volume, Task Performance, and Crew Health, Well-Being, and Safety. Mass criteria track the effect that the interior layout has on the overall mass of the habitat. Volume criteria measure the efficiency in the utilization of the interior volume and general psychological acceptability of the space. Task Performance criteria measure the impact of the layout to the productivity of crew through the impact of schedule based factors and the placement of tasks within the habitat. Finally Crew Health, Well-Being, and Safety criteria track several factors which directly impact the physiological or psychological health of the crew or pertain to contingency operations. Each of these criteria is summarized in Appendix A: Evaluation Criteria Descriptions

Table 5 in Appendix A and described in detail (including quantification methods) as shown in Appendix A: Figure 18 and Figure 19 for habitable volume and separation for privacy examples.
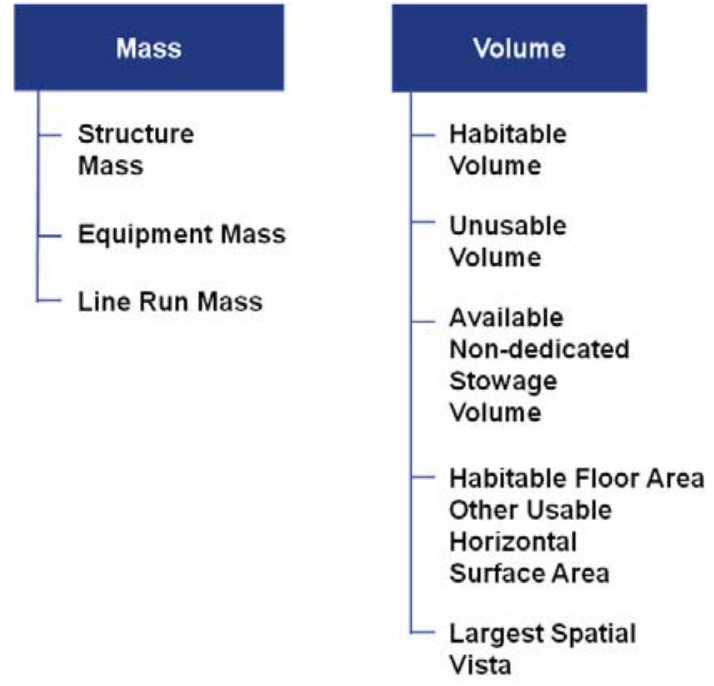

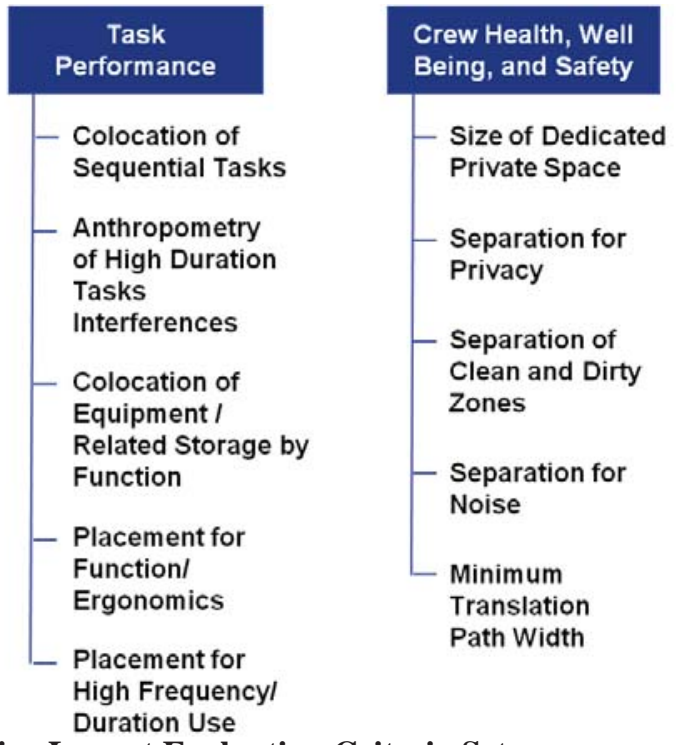

Figure 5: Comprehensive Habitat Interior Layout Evaluation Criteria Set

In the development of this set of evaluation criteria, several qualitative criteria were mapped to quantitative proxies. Review of previous evaluation methods has shown that user measurement of evaluation criteria must be reduced to ensure consistent evaluations and accelerate the evaluation process. In the creation of a fully quantitative set of evaluation criteria, analysis and reformulation of qualitative measures is needed. Figure 6 outlines the process taken to reformulate the criteria with a practical example. Spaciousness is a broad measure of the psychological acceptability of the size and shape of an interior layout. It is measured by a wide range of criteria which can loosely be split into two categories: quantitative and qualitative. The quantitative measures are easily measurable and straightforward in definition. The qualitative measures are more difficult to measure, as they deal more with perception than physical measurement. Qualitative measures can be divided into three basic categories. Semi-layout independent measures like color or a "clean look' tend to be modifiable with little to no impact on the design, layout, or size and can be removed from consideration. Designer preferences like the relative importance of each of these measures or the acceptable values of each criterion have already been discussed as weightings and utilities. 
Perception-driven measures are still dependent upon layout, but cannot be determined without user assessment of the layout in an analogous test situation. However, measurable proxy variables can be used to approximate qualitative perceptionbased criteria. For example, in Figure 6 crowdedness measures the degree to which crewmembers will feel crowded or that their tasks are impeded by the presence of other crew members. This is strongly correlated with the privacy measure which measures the extent to which crew feel that their privacy needs are met. The combination of several quantitative measures like the number of overlaps of high-frequency and high duration tasks (which use schedules and task locations to measure how often crew locations might overlap) and the width of the translation paths (which measures the ability of crew to pass by one another without intersecting) can

\section{Example: $\quad$ Spaciousness}

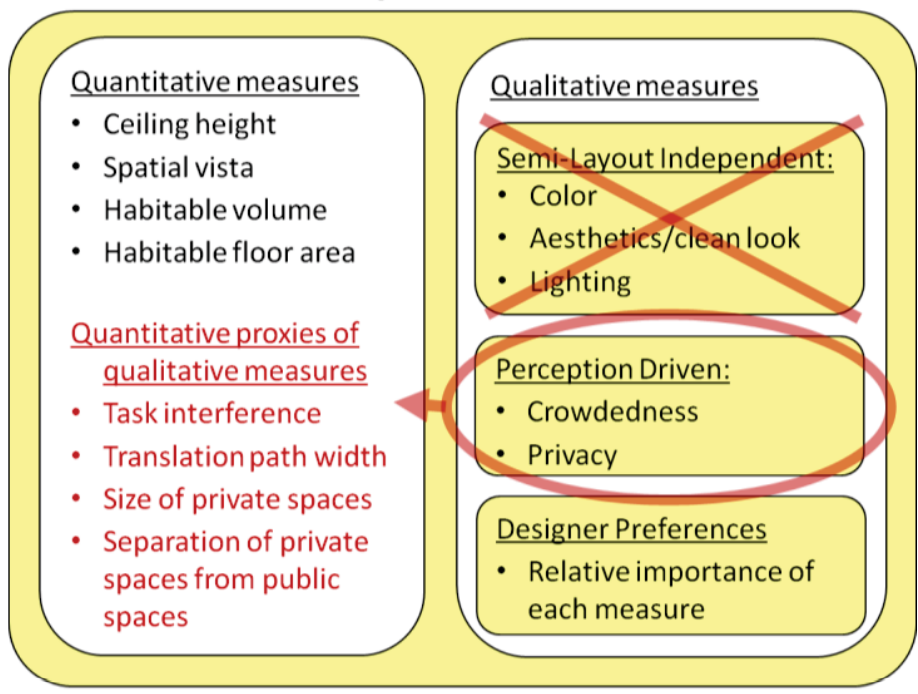

Figure 6: Mapping of Qualitative Evaluation Criteria to Quantitative Proxies approximate the potential for crowding. Similarly the size and distribution of private and public spaces can also approximate aspects of crowdedness and privacy. By mapping all qualitative perception-based measures to quantitative proxies which approximately measure the same factors, a fully quantitative criteria set can be created. Every criterion in Figure 5 can be measured directly or by some quantitative proxy. Ensuring that these criteria can be automatically measured is the focus of Section III.

\section{Objective Function}

The final step of the layout evaluation process is the assembly and calculation of a weighted, constrained multicriteria objective function from the designer preferences, evaluation criteria values, and applicable constraints. A high level description of the weighted-sum multi-criteria objective function is provided in Equation 1.

$$
Y(A)=1-\sum_{i} w_{i} U_{i}\left(X_{i}(A)\right)+\sum_{j} P_{j}(A)
$$

where: $\mathrm{Y}(\mathrm{A})=$ the aggregate value of layout $\mathrm{A}$

$\mathrm{w}_{\mathrm{i}}=$ the relative importance weighting of each criteria $\mathrm{i}$

$\mathrm{X}_{\mathrm{i}}(\mathrm{A})=$ the measured/calculated values of each criterion, $\mathrm{i}$, corresponding to the layout A (specified by locations of equipment)

$\mathrm{U}_{\mathrm{i}}\left(\mathrm{X}_{\mathrm{i}}(\mathrm{A})\right)$ = The utility function value for each criteria calculated from the measured value $\mathrm{X}_{\mathrm{i}}(\mathrm{A})$

$\mathrm{P}_{\mathrm{j}}(\mathrm{A})=$ Penalty function values to enforce constraint $\mathrm{j}$ for layout $\mathrm{A}$

$$
\text { where } P_{j}(A)=\left\{\begin{aligned}
0 & \text { if constraint is met } \\
\alpha * g j(x) 2 & \text { if the } j \text { th constraint is violated }
\end{aligned}\right.
$$

where $\mathrm{g}(\mathrm{x})$ is the amount the constraint is violated and $\alpha$ is some constant indicating how hard the constraint is to be applied

The resulting function represents an aggregate measure of the overall performance of a layout for the specific set of designer preferences. It provides a structured and quantifiable way to justify the selection of one configuration over another and provides insight into which interior design features most directly affect the 'goodness' of a configuration. Furthermore, eliminating subjectivity in the measures of layout effectiveness enables the use of a stochastic optimization method to quickly improve interior layout designs, so long as the methods used to calculate the evaluation criteria are not too time consuming. This process has been utilized on many similar problems in space layout planning ${ }^{29-31}$.

It should be noted that the quality of each evaluation greatly depends on the fidelity of the modeling and supporting data that feeds into the measured evaluation criteria values. For this paper, low-medium fidelity data is used to demonstrate the operation of the methodology. 


\section{Evaluation Criteria Quantification Methods}

Though each of the evaluation criteria shown in Figure 5 is quantifiable, that does not imply that a method exists to automatically calculate its value without user interaction. For example, habitable volume is a quantifiably measurable quantity, but the current method for measuring it from an interior layout is manual measurement using a CAD mode ${ }^{36}$. There are also several other desired characteristics of the measurement methods:

- Calculable from layout and available data, with no user interaction

- Require minimal computational time to solution

- $\quad$ Scalable to various design precisions

- $\quad$ Traceable to definitions of measures provided in references (particularly Ref. 6)

- $\quad$ Easy to setup

Several mechanisms enabling the automatic calculation of evaluation criteria values were identified through extensive investigation of related fields of study including packing/container loading problems ${ }^{37,38}$ and space layout planning $^{29-31}$. In particular, three mechanisms were identified which will enable the development of measurement methods meeting all desired characteristics: 1) collision detection, 2) grid-based numerical methods, and 3) separation/collocation matrices.

\section{A. Collision Detection}

Collision detection (also known as interference detection or contact determination) is the detection of contact, overlap, or intersection of geometries ${ }^{8}$. It is used extensively in video games, virtual prototyping, robotics, animation, and engineering simulations. In the evaluation of habitat layout alternatives it serves two purposes. First, interferences between pieces of hardware and between hardware
Test if point is in interior of an object

- Habitable volume, unusable volume, stowage volume, minimum translation path width, size of dedicated private space

Intersection point between a line and an object

- Spatial vista, minimum translation path width

\section{Collision detection between three-dimensional geometries \\ - Anthropometry of high duration tasks, \\ - Realizability check}

and pressure vessel structure must be detected to ensure that only physically realizable layouts are acceptable. Second, collision detection can be used to measure several of the volume evaluation criteria which measure volume or task performance criteria counting potential interferences between different types of objects.

To develop quantification methods for the evaluation criteria listed in Figure 5 and Appendix A, the three types of collision tests are summarized in Figure 7. Figure 7 also lists the criteria which depend upon these methods for quantification. Testing if a point is inside an object allows for the determination of sizes of open volumes when combined with numerical methods discussed in the next section. Determining the intersection point between a line and an object can be used to determine line of sight distances. Collision detection between three-dimensional geometries enables the detection of interferences between pieces of hardware or anthropometric envelopes and is critical to the calculation of the realizability constraint.

The methods for performing these collision tests are well understood and code is freely available ${ }^{38,39}$. Three figures of merit are used in the evaluation of collision detection methods: performance (i.e., run time), accuracy in detecting collisions, and ease of implementation. Based upon these figure of merit and the availability of a collision detection library in Ref. 39, the Incremental Separating Axis - Gilbert-Johnson-Keerthi (ISA-GJK) algorithm ${ }^{39,40}$ was selected for each of the types of collision detection in Figure 7. This algorithm provides fast and consistent collision detection between any two convex geometries. When a collision test between a point and hardware geometry is required, the point can be modeled as a small sphere enabling one type of collision test for all applications. This algorithm is described in detail in Ref 39.

Because numerical methods requiring several million collision detection calls are used to calculate evaluation criteria, fast and reliable collision detection is necessary. The criticality of fast collision detection tests for video game and computer animation applications has resulted in the development of many methods to speed up collision detection tests. The primary way of ensuring real-time performance of collision detection methods is to eliminate low-level tests by removing pairs of objects which clearly do not collide from consideration. This is achieved by the use of two major concepts: bounding volumes and spatial partitioning. Bounding volumes use simplified geometries to approximate more complicated objects so that expensive tests of complex objects can be avoided. Spatial 
partitioning sets up a hierarchy to reduce the number of object pairs tested based upon their location, only testing those in the same region of space. In particular, axis aligned hierarchal partitioning where each parent can have 8 children known as an octree is used in this analysis.

\section{B. Grid-Based Numerical Methods}

To eliminate CAD-based manual measurement of volumes, an automatic method of measuring and characterizing different types of volume is required. A numerical integration approach using an orthogonal Cartesian grid of discrete test points spanning the pressurized volume of the habitat, shown in Figure 8, was proposed. This method first tests points in space to determine if they are occupied by hardware or 'free'. It then applies several

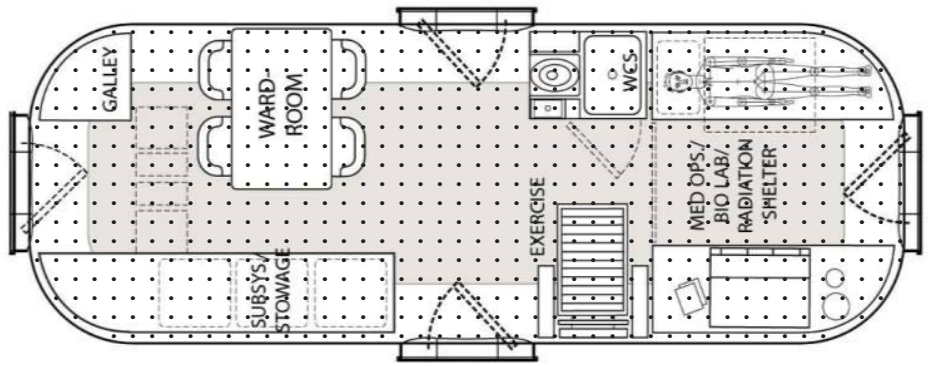

Figure 8: Orthogonal Cartesian Grid of Test Points for Iterative Evaluation Criteria Measurement Methods

Boolean collision detection tests based upon the definitions of volume-based criteria to determine if the point should count towards a particular type of volume. After characterizing all points in the pressurized volume, the total amount of a particular type of volume can then be determined by summing the points conforming to the criteria definition and using this sum with the resolution of the grid to make a numerical approximation to the volume represented by the points.

For example, a test point can be characterized as part of the habitable volume by testing whether it is within the free, accessible, and functionally usable volume. The specific Boolean tests used to determine if the point should be included in habitable volume are all "point-hardware" collision tests defined in the previous section:

- Is the point inside of the pressure vessel?

- Is the point under the floor or above the ceiling? (as represented by polyhedral geometry features)

- Is the point inside any hardware geometry?

- Is the point above reachable height? (assuming a non-microgravity environment)?

- Is the point accessible by a standing or prone astronaut? (illustrated in Figure 9)

- Is the point accessible by a reaching arm connected to a standing or prone astronaut? (illustrated in Figure 9) These points can then be summed as shown in Figure 10 for the Scenario 12.0 PCM to calculate the total habitable volume. A similar set of test points can be defined for all of the volume-related evaluation criteria.
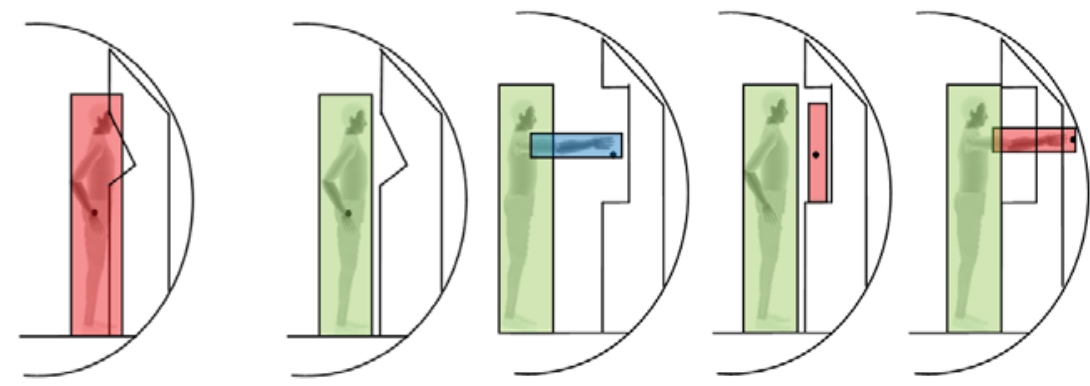

Figure 9: Illustration of Accessible Space for Habitable Volume Calculation

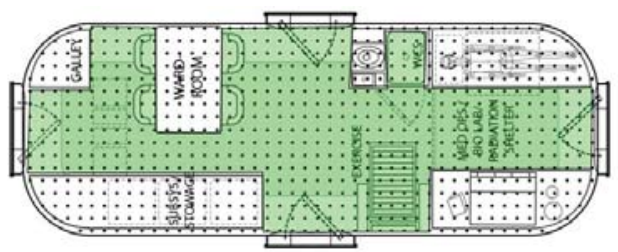

\begin{tabular}{|c|c|c|}
\hline Resolution (m) & $\begin{array}{c}\text { Number of } \\
\text { points }\end{array}$ & $\begin{array}{c}\text { Habitable } \\
\text { Volume } \mathbf{( m}^{\mathbf{3}} \text { ) }\end{array}$ \\
\hline 0.10 & 33600 & 33.6 \\
\hline 0.05 & 269600 & 33.7 \\
\hline 0.01 & 33720000 & 33.7 \\
\hline
\end{tabular}

Figure 10: Calculation of Scenario 12.0 PCM Habitable Volume

The discrete grid of points can also be used for iterative methods of defining length-based evaluation criteria, particularly spatial vista and minimum aisle width. Spatial vista measures the maximum amount of volume that a crewmember can see within the habitat as shown in Figure 11. The point of origin from which the viewer's perspective originates is important to the find the maximum possible volume. The discrete points in the grid can be 
used in combination with an optimization method to converge to the point of view measuring the maximum spatial vista. Similarly, to quantify the minimum translation path width, points of origin must be on the translation path, which changes with every layout. The grid of points can be used with a robot path planning algorithm like A* or Djkstra's algorithm to construct a translation path from the available points. Then these identified points can be used as the points of origin for the measurement of translation path width as shown in Figure 12.

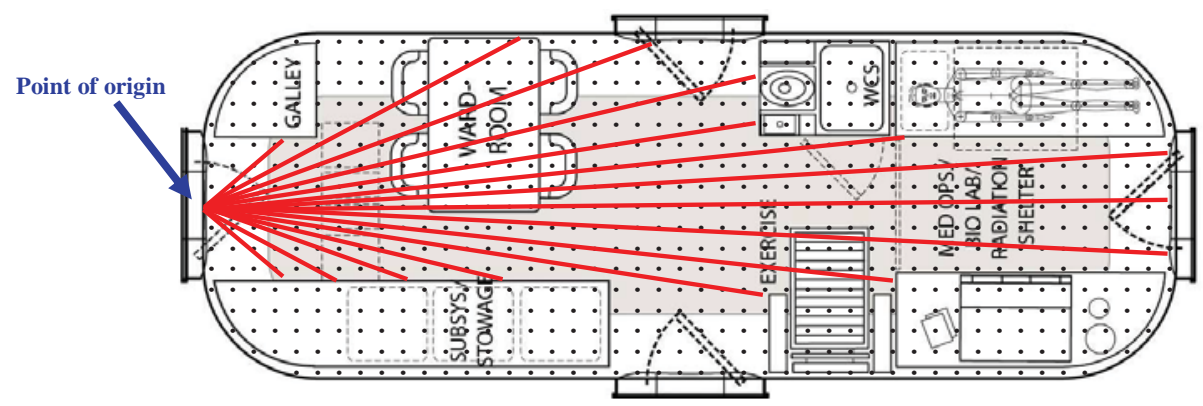

Figure 11: Use of Discrete Grid-based Iterative Method to Calculate Spatial Vista

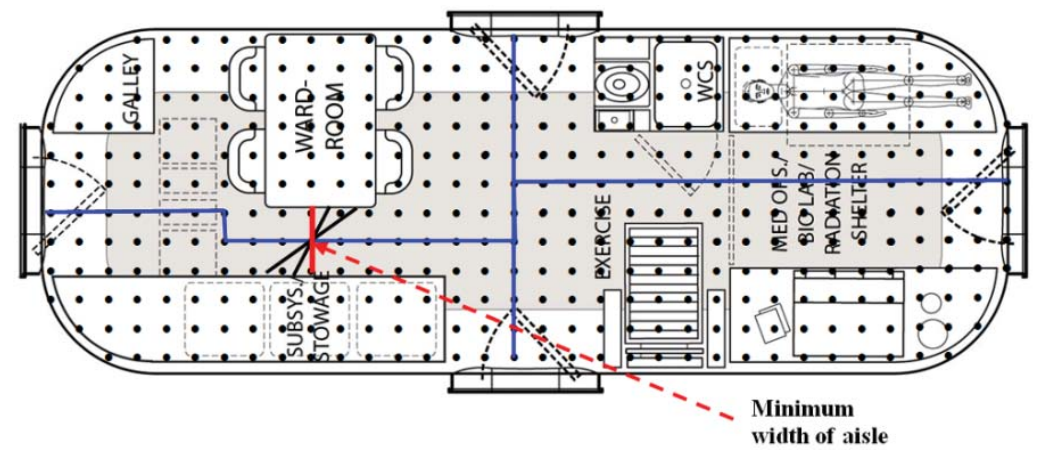

Figure 12: Use of Grid-based Iterative Methods to Measure Minimum Translation Path Width

It is important to note that for even moderately sized habitats, the assumed resolution of the grid necessary to get accurate measurements may include a computationally prohibitive amount of points. For example, a habitat with $3 \mathrm{~m}$ diameter and $7 \mathrm{~m}$ length may take as many as 60 million points to characterize the space. Storing data such as the location and exact characteristics of each of these points would be difficult. Two methods are taken to prevent this problem:

1. Reduced grid resolution to reduce the number of points which must be tracked. This is particularly important in finding the minimum translation path width which must keep track of the location of points in the translation path.

2. Additive calculation with no data can be used to eliminate the need for data storage. For example, when calculating habitable volume, the number of points passing each of the Boolean tests can be simply be counted using a running counter to determine the volume.

\section{Separation-Colocation Matrices}

Several evaluation criteria measure how well the functional relationships between systems are accommodated by their location. This can also be thought of as the degree to which conflicting or complementary hardware are separated or collocated, respectively. In spacecraft design and terrestrial architecture this is known as a functional relationships analysis ${ }^{24,26}$.

A calculation method utilizing two types of matrices has been identified in literature ${ }^{24}$ :

1. Matrices capturing the functional relationships between crew tasks are drawn from station crew schedules and crew preference elicitation. Mapping these tasks to pieces of hardware provides guidance as to whether hardware should be separated or collocated based upon specific criteria such as noise, hygiene, etc.

2. Matrices of the distances between objects can be defined based upon the layout and combined with the function relationships matrices to derive a measure of how successful the layout is at accommodating these functions. The distances included in these matrices need not be restricted to Euclidean distances. Some 
criteria desire visual separation or hygiene separation which can be augmented by partitions while others dealing with the length of shared consumable lines which run along the pressure vessel of the habitat behind equipment are best measured with some cylindrical mapping of Manhattan distance.

The structures of these matrices are shown in Figure 13. In order to derive a single measure of the overall effectiveness of a layout to capture these relationships, the Euclidean norm of the Hadamard Product (entry-wise product) of the Relationships (R) and Distance (D) matrices was used. $\left\|[R \cdot D]_{\mathrm{ij}}\right\|$. While this quantity is not physically interpretable as any measurement, it can be compared to the range of possible values of this product determined by a design space exploration of the distance matrix for the specified pressure vessel geometry to gage its performance against possible values.
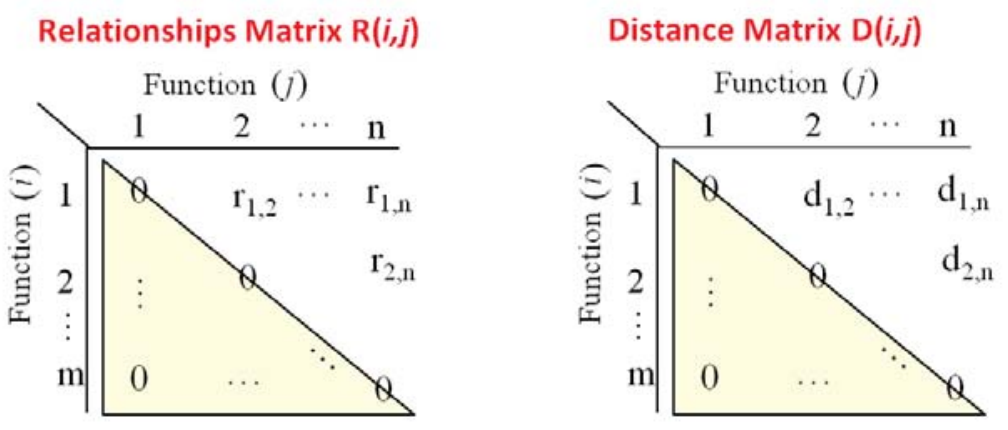

Figure 13: Functional Relationships and Distance Matrices

Detailed automatically calculable algorithms have been created for each of the evaluation criteria in Figure 5 by implementing combinations of each of the three enabling mechanisms with logical definition-derived measurements. These algorithms are designed for implementation in an object oriented programming language compatible with basic geometry modeling. A high level comparison of the quantification methods used in the upcoming example problem can be found in Appendix A.

\section{Software Tool Implementation}

C++ and OpenGL were chosen to implement the method described above. These languages were primarily chosen because of the availability of collision detection libraries and the ability to generate a transferrable executable file to facilitate sharing. The code operates in the following order. First, the problem description information, such as the size of the habitat, is defined. Then, information about the pieces of hardware to be included in the layout is read from .csv input files. This includes the geometry of these objects, geometry of reserved volume to interact with them, mass, volume, what tasks are associated with hardware, etc. The positions of hardware may be specified in this input file to model one layout or they may be separately defined in another file to create a population of layouts. All of the information about the objects is stored in "structs", which allow for colocation and facilitated access of geometry, position, and functional relationship data. Other input files capture the utility functions and criteria weightings which are determined from expert elicitation over the course of a few hours, but these inputs could also be pulled from libraries of previous runs in future code implementations.

After all input information is captured; the evaluation criteria are calculated from the hardware, layout, and relationship data. Iterative methods with several Boolean tests are used to measure volumes, numbers of interferences, and priority placement of hardware. For other criteria, simple analytical calculations are used to obtain values. Values for

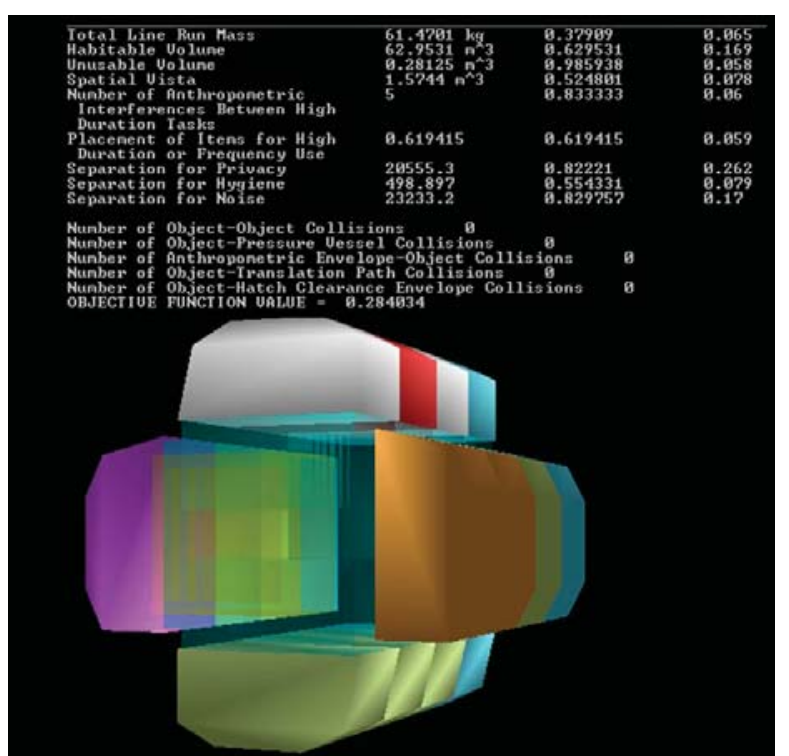

Figure 14: OpenGL Output of Layout Evaluation Tool 
these criteria are combined with input utility function parameters to map these measured values to the utility values, which are then combined with weightings in a weighted sum to obtain the unconstrained performance of each layout. Then a set of collision tests are used to calculate constraint violations. Violated constraints are then added to the unconstrained objective function values to obtain the constrained performance of a layout. Images of the layout are then rendered using the OpenGL visualization capability as shown in Figure 14, using transparent blue and yellow boxes to track the reserved volumes and translation paths.

In addition to evaluating single layouts, the tool is set up to generate new layout matrices and evaluate multiple alternative layouts. These populations of layouts are generated using Particle Swarm Optimization (PSO) which is a stochastic optimization algorithm which has been successful for similar problems in space layout planning ${ }^{41-43}$. This algorithm alters the layout matrices based upon global and personal best objective function values in previous iterations. Alternative methods derived from PSO are being investigated to achieve better optimization performance.

\section{Example Problem: 180 day Cis-Lunar Habitat}

In order to demonstrate this tool and drive out necessary improvements, the following example design problem was proposed:

Evaluate the layout performance of a cis-lunar habitat designed to sustain four crew for 180 days in deep space with an entry capsule. Assume the spacecraft is 4.5 meters in diameter and 6 meters long with one hatch on an endcap. Assume a pre-defined set of rack-based hardware with standard anthropometric use envelopes, which is to be packaged within this habitat while maintaining a central translation path corridor.

The topology of the baseline layout to be evaluated is shown in Figure 15. The positions of each of the subsystems are indicated by the rows which correspond to the standard wall, ceiling, and floor rack locations. The functions in this module (long duration accommodations and closed loop life support) are focused on increasing crewed duration while functions in attached modules are focused on increased exploration and science.

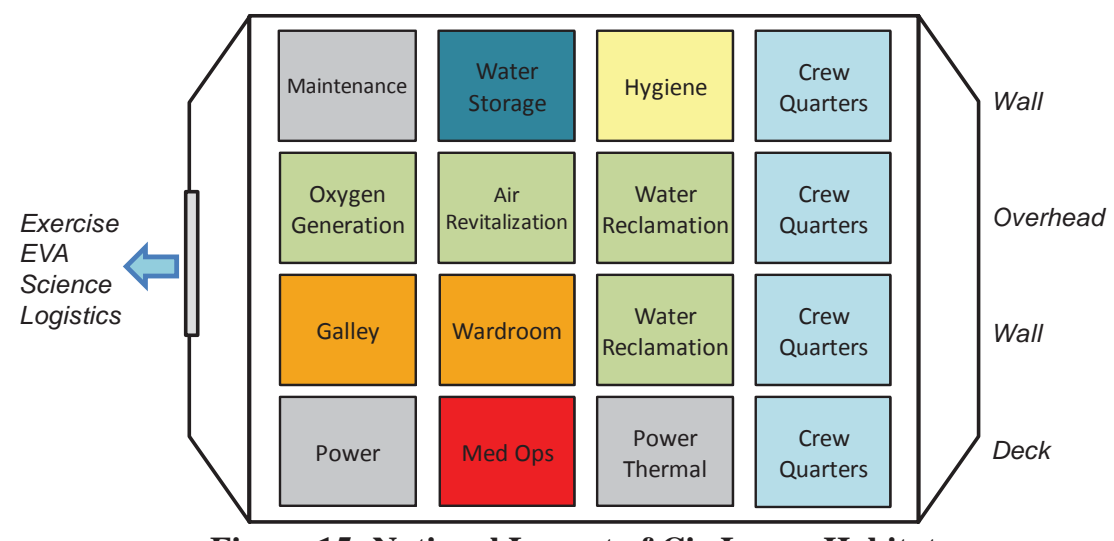

Figure 15: Notional Layout of Cis-Lunar Habitat

The evaluation began with the definition of designer preferences. First, the evaluation criteria are weighted based upon the design problem context. Larry Toups, an architect and habitat design expert from NASA Johnson Space Center, performed pair-wise comparisons of the evaluation criteria which were used to create the prioritized list shown in Figure 16 where the weightings are expressed in percentages. The criteria used to evaluate the cis-lunar habitat are reduced to those which make up the top $80 \%$ of the weightings. The methods for measuring these criteria are included in Appendix A. Linear utility functions are assumed for these criteria with value ranges indicated in Table 2. Habitable volume ranges are set based upon human spaceflight design documents, but could be modified if additional living volumes are assumed to be provided by additional modules. The other criteria value ranges are assumptions based upon values seen in a quick analysis of possible layouts.

Table 2: Utility Functions for Cis-Lunar Habitat Evaluation Criteria

\begin{tabular}{|l|c|c|}
\hline Evaluation Criteria & Value for U=0 & Value for U=1 \\
\hline Plumbing/Electric Line Run Masses & $1 \mathrm{~kg}$ & $100 \mathrm{~kg}$ \\
\hline Habitable Volume & $0 \mathrm{~m} 3$ & $100 \mathrm{~m} 3$ \\
\hline Unusable Volume & $20 \mathrm{~m} 3$ & $0 \mathrm{~m} 3$ \\
\hline Largest Spatial Vista & $0 \mathrm{~m} 3$ & $3 \mathrm{~m} 3$ \\
\hline Anthropometry of High Duration Tasks Interferences & 30 interferences & 0 interferences \\
\hline Placement for High Frequency/Duration Use & 0 & 1 \\
\hline Separation for Privacy & 0 & 25000 \\
\hline Separation of Clean and Dirty Zones & 0 & 900 \\
\hline Separation for Noise & 0 & 28000 \\
\hline
\end{tabular}




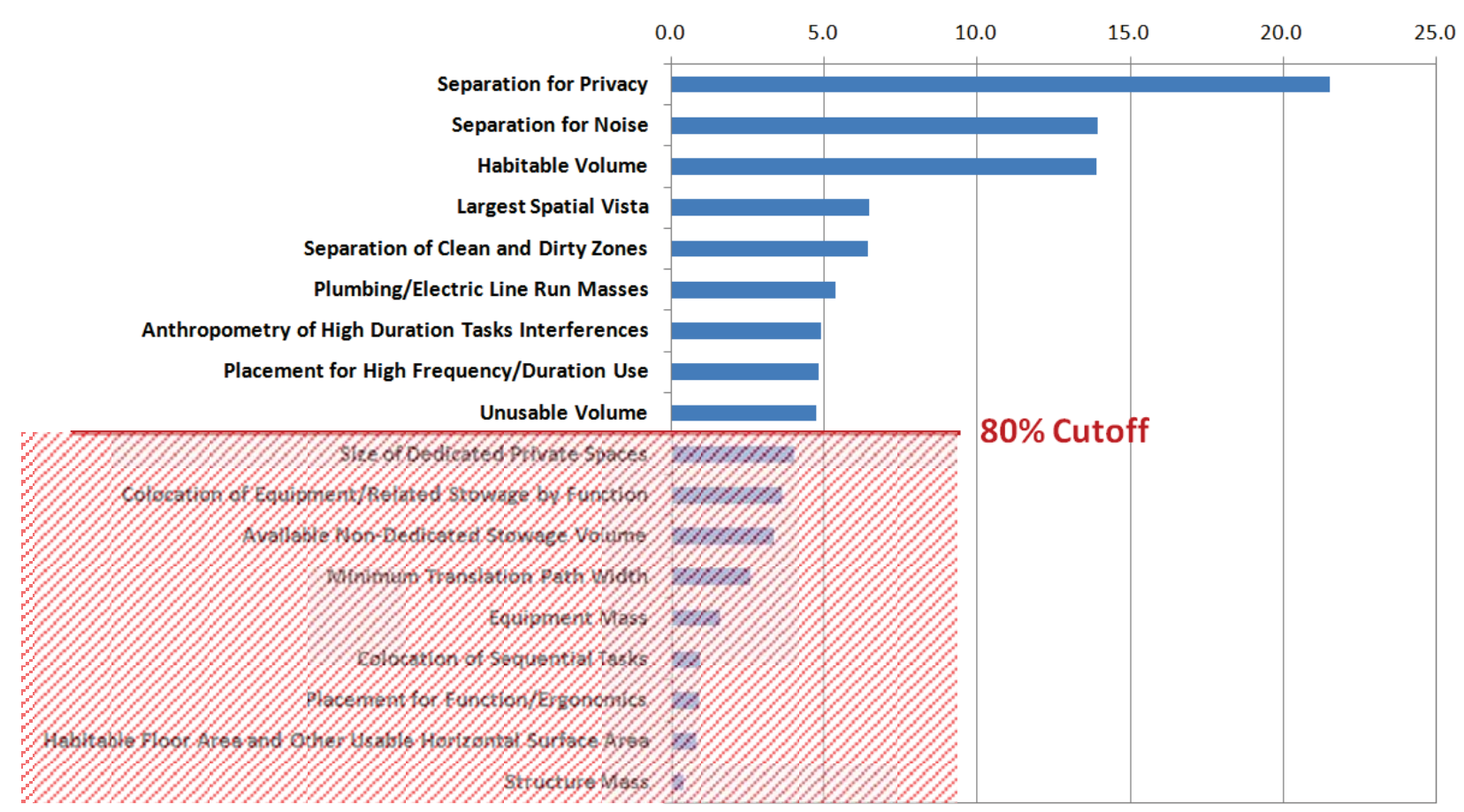

Figure 16: Prioritized Weighting of Evaluation Criteria

For simplicity, the only constraints chosen for this example problem are hardware interferences ("object-object" and "object-wall") and clearance checks ("anthropometric envelope-object", "object-translation path", and "objecthatch clearance envelope"). Constraints are implemented based upon the number of interferences observed. For example, some multiple of the number of anthropometric reserved volume and object collisions is added to the unconstrained objective function to decrease acceptability. These tests are implemented with the same ISA-GJK collision detection test used for the evaluation criteria quantification methods.

\section{Results:}

Evaluation of the baseline layout yielded the results shown in Table 3. Assuming the utility ranges are appropriate, the resultant utilities indicate that all evaluation criteria perform moderately well except line run mass, spatial vista, and separation of clean and dirty tasks. The aggregate value shows that this layout performs moderately well (minimum values are preferred). Because of the somewhat arbitrary selection of value ranges in the utility function definitions, few definitive conclusions can be drawn about whether the performance of the layout for a specific criterion is acceptable or not. However, comparing multiple layouts with the same set of assumptions and designer preferences allows for the determination of a relative acceptability of the layouts which can be used to guide iterations towards an optimal solution.

For example, trading the rack based locations of a few subsystems as shown in Figure 17 yields the results shown in Table 4. This layout performs better on line run mass and anthropometric interferences, but at the expense of the separation-colocation criteria and placement for high duration/frequency of use. Because of the relatively high weighting of these separation-colocation criteria, this significantly decreases the overall acceptability of this layout. 
Table 3: Baseline Layout Objective Function Calculation

\begin{tabular}{|c|c|c|c|}
\hline Evaluation Criteria & Measured Value & Utility & Weightings \\
\hline Total Line Run Mass & $61.5 \mathrm{~kg}$ & 0.38 & 0.065 \\
\hline Habitable Volume & $63.0 \mathrm{~m}^{3}$ & 0.63 & 0.169 \\
\hline Unusable Volume & $0.28 \mathrm{~m}^{3}$ & 0.99 & 0.058 \\
\hline Spatial Vista & $1.57 \mathrm{~m}^{3}$ & 0.52 & 0.078 \\
\hline Anthropometric Interferences between High Duration Tasks & 5 & 0.83 & 0.060 \\
\hline Placement of Items for High Duration /Frequency Use & 0.62 & 0.62 & 0.059 \\
\hline Separation for Privacy & 20555 & 0.82 & 0.262 \\
\hline Separation of Clean and Dirty Spaces & 498.9 & 0.55 & 0.079 \\
\hline Separation for Noise & 23233 & 0.83 & 0.170 \\
\hline Constraints & Measured Value & & \\
\hline Number of Object-Object Collisions & 0 & & \\
\hline Number of Object-Pressure Vessel Collisions & 0 & & \\
\hline Number of Anthropometric Envelope-Object Collisions & 0 & & \\
\hline Number of Object-Translation Path Collisions & 0 & & \\
\hline Number of Object-Hatch Clearance Envelope Collisions & 0 & & \\
\hline OBJECTIVE FUNCTION VALUE & 0.28 & & \\
\hline
\end{tabular}

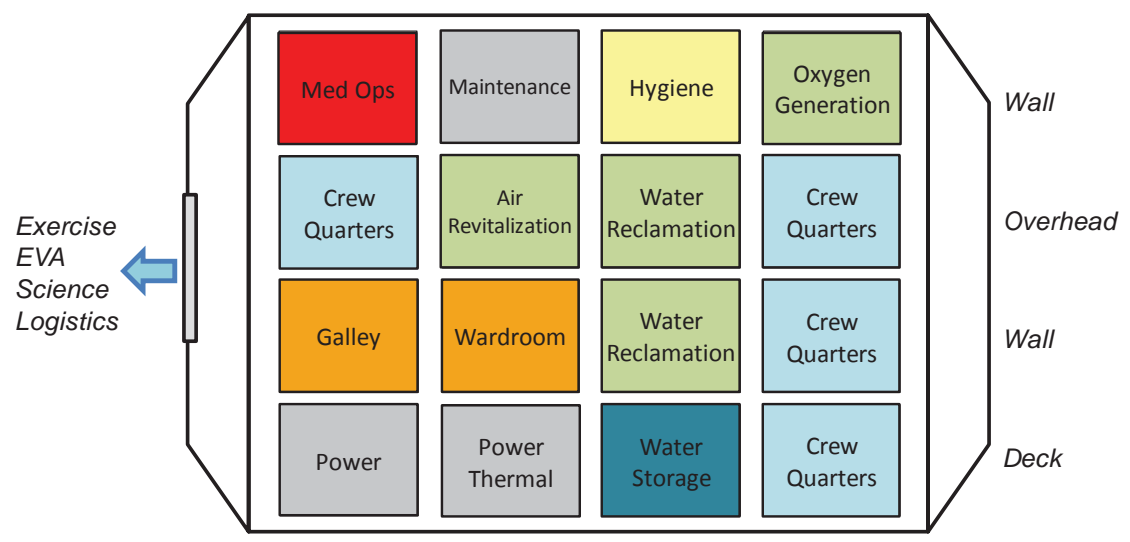

Figure 17: Alternate Layout of Cis-Lunar Habitat

Table 4: Alternative Layout Objective Function Calculation

\begin{tabular}{|l|c|c|c|}
\hline Evaluation Criteria & Measured Value & Utility & Weightings \\
\hline Total Line Run Mass & $44.6 \mathrm{~kg}$ & 0.55 & 0.065 \\
\hline Habitable Volume & $63.0 \mathrm{~m}^{3}$ & 0.63 & 0.169 \\
\hline Unusable Volume & $0.31 \mathrm{~m}^{3}$ & 0.98 & 0.058 \\
\hline Spatial Vista & $1.57 \mathrm{~m}^{3}$ & 0.52 & 0.078 \\
\hline Anthropometric Interferences between High Duration Tasks & 3 & 0.9 & 0.060 \\
\hline Placement of Items for High Duration /Frequency Use & 0.51 & 0.51 & 0.059 \\
\hline Separation for Privacy & 13431 & 0.54 & 0.262 \\
\hline Separation of Clean and Dirty Spaces & 313 & 0.35 & 0.079 \\
\hline Separation for Noise & 14351 & 0.51 & 0.170 \\
\hline Constraints & Measured Value & & \\
\hline Number of Object-Object Collisions & 0 & & \\
\hline Number of Object-Pressure Vessel Collisions & 0 & & \\
\hline Number of Anthropometric Envelope-Object Collisions & 0 & & \\
\hline Number of Object-Translation Path Collisions & 0 & & \\
\hline Number of Object-Hatch Clearance Envelope Collisions & 0 & & \\
\hline OBJECTIVE FUNCTION VALUE & $\mathbf{0 . 4 2}$ & & \\
\hline
\end{tabular}




\section{Conclusions and Future Work}

The proposed evaluation method has been fully successful in delivering structured, fast, and complete habitat interior evaluations useful for habitation design community and any other community designing highly constrained, highly integrated interiors. The critical contributions of this research are the development of a comprehensive, measurable set of evaluation criteria which capture a full range of engineering (mass and volume) and habitability (quality of volume, functionality, safety, etc.) concerns and the development of automatic methods to measure them. These quantification methods substantially increase the state of the art in evaluation times (each layout evaluation requiring numerical volume estimations can be performed in about a second, while evaluations not requiring numerical methods are several orders of magnitude faster). These fast criteria measurement methods substantially increase the information available at the conceptual design phase. They provide a structured and quantifiable way to justify the selection of one configuration over another for a given set of objective preferences, which will enable trades to be performed identifying the architectural elements which most directly affect the 'goodness' of a configuration. They will also increase available knowledge of the mechanisms of designing utility into interior designs and enable designers to trade the shapes/sizes of hardware and pressure vessels to suit the objectives dictated by the mission.

The main focus of future work is to enable the automated generation of layout interior designs by continuing to mature the implementation of the particle swarm optimizer to deal with the discontinuous, multi-modal nature of the interior layout design space. Initial results utilizing the particle swarm optimizer tend to be dominated by constraints. Currently, modified particle swarm methods are being investigated to deal with these challenges.

The addition of fast interior layout generation and optimization to the evaluation tool will also:

- Facilitate designer capabilities to respond to requirements changes, investigate 'what if' scenarios, and ensure good integration with other elements of the system architecture as the concept is developed (lander, propulsive stages, and launch vehicles); all of which are characteristic of conceptual design process of exploration missions.

- Enable early representation of the layout dependent habitat design concerns affecting mission design or the design to project management or other element design teams during conceptual design.

- Provide more complete coverage and documentation of the configuration design space, leading to the discovery of better alternatives or the identification of important features which improve the design.

- Enable designers to provide justified 'push-backs' on limiting constraints or requirements for the first time in literature.

- Could reduce design time and resources to save in development costs both directly (less spent - more saved) and indirectly (better configurations require less expensive design changes later in the product lifecycle).

- Enable trade studies, including understanding the impact to the habitat configuration when trading various requirements, managerial preferences (weightings), choice of subsystems, and different component geometry shapes. Trades such as these can be used to expedite knowledge normally discovered in later design stages to the conceptual design phase.

\section{References}

${ }^{1}$ Howe, A. S. and Sherwood, B., Out of this World: A New Field of Space Architecture, AIAA, 2009.

${ }^{2}$ Kennedy, Kriss, "The Vernacular of Space Architecture", AIAA 2002-6102, AIAA Space Architecture Symposium, Houston, TX, 2002.

${ }^{3}$ Simon, Matthew, and Wilhite, Alan W., "Systems Level Evaluation of Space and Planetary Habitat Interior Layouts", AIAA-2010-8220, Modeling and Simulation Technologies Conference, Toronto, ON, Canada, 2010.

${ }^{4}$ NASA, “SP-2007-6105: NASA Systems Engineering Handbook”, NASA, 2007.

${ }^{5}$ Kennedy, K., Toups, L., Rudisill, M., "Constellation Architecture Team-Lunar Scenario 12.0 Habitation Overview", NASA, JSC-CN-19097, 2009.

${ }^{6}$ NASA, "Human Integration Design Handbook", NASA/SP-2010-3407, 2010.

${ }^{7}$ Larson, W. J. and Pranke, L. K., Human Spaceflight: Mission Analysis and Design, McGraw Hill Companies Inc., New York, NY, 1999.

${ }^{8}$ Ericson, C., Real Time Collision Detection, Elsevier, San Francisco, CA, 2008.

${ }^{9}$ Saaty, T. L., The Analytic Hierarchy Process, McGraw-Hill, New York, 1980.

${ }^{10}$ Smith, G. Logical Decision for Windows: Users Manual. Fairfax, VA. http://www.logicaldecisions.com, 2007. 
${ }^{11}$ National Aeronautics and Space Administration. Man-Systems Integration Standards, NASA STD-3000, Revision B. Houston: Johnson Space Center, 1995.

${ }^{12}$ Vanderplaats, G.N. Numerical Optimization Techniques for Engineering Design, McGraw-Hill Book Company, New York, 1984.

${ }^{13}$ SICSA, "Report IV: Lunar Module Habitability and Interior Outfitting Considerations and Concepts", SICSA Report, University of Houston, Houston, TX, 2009.

${ }^{14}$ Celentano, J. T.; Amorelli, D.; Freeman, G. G., "Establishing a Habitability Index for Space Stations and Planetary Bases", AIAA 63-139, AIAA/ASMA Manned Space Laboratory Conference, Los Angeles, California, USA, 1963.

${ }^{15}$ Cohen, M., "Habitat Multivariate Design Model Pilot Study", SAE 2004-01-2366, 34th International Conference on Environmental Systems (ICES), Colorado Springs, CO, July 2004.

${ }^{16}$ NASA, "Guideline and Capabilities for Designing Human Missions", NASA-TM-2003-210785, NASA, January, 2003.

${ }^{17}$ Rysavy, G. and Council, C. D., "Architecture and Environment: Basic Tools of Habitability in Space System Design”, AIAA-1971-879, AIAA/ASMA Weightlessness and Artificial Gravity Meeting, Williamsburg, VA, 1971.

${ }^{18}$ Jones, Walton, "Habitability in Long-duration Space Missions", Environmental Biology and Medicine, Vol.2, p 29-45, 1973.

${ }^{19}$ Cohen, M. M. "Designing Space Habitats for Human Productivity", SAE 901204. In, SAE Transactions, Journal of Aerospace, vol. 99, sec. 1, part 1, p. 352-364, 1990.

${ }^{20}$ Whitmore, M., McQuilkin, M., Woolford, B., "Habitability and Performance Issues for Long Duration Space Flights", NASA/CR-97-112974, NASA, 1997.

${ }^{21}$ Adams, C., "Four Legs in the Morning: Issues in Crew-Quarter Design for Long-Duration Space Facilities (SAE 981794)". 28th International Conference on Environmental Systems (ICES), Danvers, Massachusetts, USA, 13-16 July 1998.

${ }^{22}$ Adams, C., McCurdy, M., "Habitability in Advanced Space Mission Design, Part Two: Evaluation of Habitation Elements." In S. Johnson, K. Chua, R. Galloway, P. Richter (Eds.), Space 2000 (p. 72-88). 7th International Conference and Exposition on Engineering, Construction, Operations, and Business in Space, Albuquerque, New Mexico, USA, 27 February - 2 March 2000.

${ }^{23}$ Osburg, J., "An Interdisciplinary Approach to the Conceptual Design of Inhabited Space Systems", Dissertation, University of Stuttgart, Germany, 2002.

${ }^{24}$ Tullis, T. and Bied, B., "Space Station Functional Relationships Analysis Final Technical Report, NASA-CR177497," NASA, 1988.

${ }^{25}$ Nixon, D., "Space Station Group Activities Habitability Module Study", NASA CR-4010, NASA, 1986.

${ }^{26}$ Fitts, D. J., "International Space Station (ISS) Internal Volume Configuration (IVC)", AIAA 2002-6114, 1st Space Architecture Symposium (SAS 2002), Houston, Texas, USA, 10-11 October 2002.

${ }^{27}$ Eckart, P., Spaceflight Life Support and Biospherics, Kluwer Academic Publishers. Dordrecht, The Netherlands, and Microcosm Inc. Torrance, CA, 1996.

${ }^{28}$ Duerk, D. P., Architectural Programming: Information Management for Design, New York: Van Nostrand Reinhold, 119-150, 210-218, 1993.

${ }^{29}$ Kalay, E.Y., Architecture's New Media. Principles, Theories, and Methods of Computer-Aided Design. The MIT Press, Cambridge, Massachusetts. 2004.

${ }^{30}$ Lobos, D., Donath, D., "The Problem of Space Layout in Architecture: A Survey and Reflections", arquiteturarevista - Vol. 6, n² 2:136-161, July to December, 2010.

${ }^{31}$ Homayouni, H., "A Literature Review of Computational Approaches to Space Layout Planning", Final Paper, Arch 588 Research Practice, University of Washington, Fall 2006.

${ }^{32}$ Di Capua, M., Mirvis, A., Medina, O., and Akin, D., "Minimum Functionality Lunar Habitat Element Design: Requirements and Definition of an Initial Human Establishment on the Moon," SAE Int. J. Aerosp. 4(1):108129, 2011, doi:10.4271/2009-01-2369.

${ }^{33}$ SICSA, "Report 1: Figure of Merit Criteria for Evaluating and Selecting Lunar Habitat module Concepts", SICSA Report, University of Houston, Houston, TX, 2008.

${ }^{34}$ Wise, J. A. "The Quantitative Modeling of Human Spatial Habitability". NASA CR-1797-16. NASA Ames Research Center, Moffett Field, CA, USA, 1985.

${ }^{35}$ NASA, SSP 50005: International Space Station Flight Crew Integration Standards, NASA, 1999. 
${ }^{36}$ Szabo, R.; Kallay, A.; Twyford, E.; Maida, J., "The Human as a System - Monitoring Spacecraft Net Habitable Volume throughout the Design Lifecycle", Human Factors and Ergonomics Society 51st Annual Meeting, Baltimore, MD, Oct. 2007.

${ }^{37}$ Dyckhoff, H., A typology of cutting and packing problems. European Journal of Operational Research, 44:145159, 1990.

${ }^{38}$ Wäscher, G., Haußner, H., Schumann, H., An improved typology of cutting and packing problems. European Journal of Operational Research, 183:1109-1130, 2007.

${ }^{39}$ van den Bergen, Gino, Collision Detection in Interactive 3D Environments (The Morgan Kaufmann Series in Interactive 3D Technology). Morgan Kaufmann, October 2003.

${ }^{40}$ Gilbert, E. G., Johnson, D. W., Keerthi, S. S., "A Fast Procedure for Computing the Distance between Complex Objects in Three-Dimensional Space", IEEE Journal of Robotics and Automation, vol. 4, no. 2, 1988.

${ }^{41}$ Önüt, S., Tuzkaya, U. R., Doğaç, B., "A particle swarm optimization algorithm for the multiple-level warehouse layout design problem," Computers \& Industrial Engineering, Volume 54, Issue 4, Pages 783-799, May 2008.

${ }^{42}$ Cheng, M.Y., Lien, L.C.: A Hybrid Swarm Intelligence Based Particle Bee Algorithm For Benchmark Functions And Construction Site Layout Optimization. In: Proceedings of the 28th ISARC, Seoul, pp. 898-904, 2011.

${ }^{43}$ Ning, L., Fe, L., Debao, S., Chang, H., "Particle Swarm Optimization for Constrained Layout Optimization," Proceedings of the 5th World Congress on Intelligent Control and Automation, June 15-19, Hangzhou. P.R. China, 2004. 
Table 5: Evaluation Criteria Descriptions

\begin{tabular}{|c|c|}
\hline & Description \\
\hline \multicolumn{2}{|l|}{ Mass } \\
\hline Structure Mass & $\begin{array}{l}\text { The mass of primary and secondary structures which vary dependent on the configuration. } \\
\text { This includes the pressure vessel, launch integration structure, hatches, windows, walls, } \\
\text { floors, ceilings, and support mass for equipment. }\end{array}$ \\
\hline Equipment Mass & $\begin{array}{l}\text { The mass of the ECLSS, TCS, Power Distribution, Stowage, Crew Accommodations, } \\
\text { Logistics, Avionics, etc equipment. Does not include power and consumable distribution } \\
\text { lines. }\end{array}$ \\
\hline $\begin{array}{l}\text { Plumbing/Electric Line } \\
\text { Run Masses }\end{array}$ & $\begin{array}{l}\text { The mass of power distribution, atmosphere distribution, vacuum, and various water } \\
\text { distribution lines based upon the placement of interior objects. }\end{array}$ \\
\hline \multicolumn{2}{|l|}{ Volume } \\
\hline Habitable Volume & $\begin{array}{l}\text { The free, pressurized volume, excluding the space required for subsystems, structural } \\
\text { elements, stowage, outfitting, accommodations, and structural Inefficiencies (nooks and } \\
\text { crannies). It is literally the space livable, accessible, and functionally usable to crew. } \\
\text { (referred to as Net Habitable by JSC) }\end{array}$ \\
\hline Unusable Volume & $\begin{array}{l}\text { The inaccessible volume and structural inefficiencies caused by the particular packing } \\
\text { strategy. Also not usable for stowage) }\end{array}$ \\
\hline $\begin{array}{l}\text { Available Non-Dedicated } \\
\text { Stowage Volume }\end{array}$ & $\begin{array}{l}\text { The available space for the storage of goods within the free volume outside of translation } \\
\text { paths and anthropometric envelopes. }\end{array}$ \\
\hline $\begin{array}{l}\text { Habitable Floor Area and } \\
\text { Other Usable Horizontal } \\
\text { Surface Area }\end{array}$ & $\begin{array}{l}\text { The floor area available for crew movement (often indicating anthropometrically } \\
\text { accessible floor area by a standing astronaut (excludes skinny spaces, space behind racks, } \\
\text { under beds, under desks, etc.)) and the area of horizontal surfaces occupied by crew } \\
\text { including desks, tables, work counters, shelves, beds, and chairs. }\end{array}$ \\
\hline Largest Spatial Vista & $\begin{array}{l}\text { The maximum volume swept by the eye of a crew member. A measure of spaciousness } \\
\text { and psychological/physiological acceptability of the environment. Analogous to maximum } \\
\text { contiguous line of sight and contiguous field of view. }\end{array}$ \\
\hline \multicolumn{2}{|l|}{ Task Performance } \\
\hline $\begin{array}{l}\text { Colocation of Sequential } \\
\text { Tasks }\end{array}$ & $\begin{array}{l}\text { The degree of colocation of tasks which are sequential (according to analogous crew } \\
\text { schedules). A measure of the overall minimized required crew translation distances } \\
\text { throughout an interior. }\end{array}$ \\
\hline $\begin{array}{l}\text { Anthropometry of High } \\
\text { Duration Tasks } \\
\text { Interferences }\end{array}$ & $\begin{array}{l}\text { The number of long duration tasks whose anthropometric volumes interfere with either the } \\
\text { anthropometric volumes of other high duration tasks, translation paths, or hatch clearance } \\
\text { areas. }\end{array}$ \\
\hline $\begin{array}{l}\text { Colocation of Equipment } \\
\text { by Function }\end{array}$ & $\begin{array}{l}\text { The degree of grouping of equipment and components based upon the function or task } \\
\text { they belong to. }\end{array}$ \\
\hline $\begin{array}{l}\text { Placement for } \\
\text { Function/Ergonomics }\end{array}$ & $\begin{array}{l}\text { A measure of the displacement of equipment from the location required by its function or } \\
\text { ergonomic operation (e.g. a desk in a gravity environment should be } \sim 36 \text { in from the } \\
\text { floor). }\end{array}$ \\
\hline $\begin{array}{l}\text { Placement for High } \\
\text { Frequency/Duration Use }\end{array}$ & $\begin{array}{l}\text { A measure of the displacement of 'high frequency/duration of use' equipment from the } \\
\text { 'prime real estate' locations for human interaction (along wall, away from hatches, waist to } \\
\text { eye level) }\end{array}$ \\
\hline \multicolumn{2}{|c|}{ Crew Health, Well Being, and Safety } \\
\hline Size of Private Spaces & $\begin{array}{l}\text { The size of the crew quarters or waste and hygiene closet or other space designated as } \\
\text { private. }\end{array}$ \\
\hline Separation for Privacy & $\begin{array}{l}\text { The degree of separation between public and private areas, such as the crew quarters and } \\
\text { the wardroom }\end{array}$ \\
\hline $\begin{array}{l}\text { Separation of Clean and } \\
\text { Dirty Zones }\end{array}$ & $\begin{array}{l}\text { The degree of separation between clean and dirty areas, such as crew quarters and hygiene } \\
\text { area }\end{array}$ \\
\hline Separation for Noise & $\begin{array}{l}\text { The degree of separation between noisy and quiet areas, such as crew quarters and the } \\
\text { wardroom area }\end{array}$ \\
\hline $\begin{array}{l}\text { Minimum Translation } \\
\text { Path Width }\end{array}$ & The minimum width along the path which allows access to each hatch and subsystems \\
\hline
\end{tabular}




\section{Habitable Volume (Quantifiable)}

- Inputs: Pressure vessel geometry, interior subsystems geometries and locations, astronaut anthropometric dimensions

- Definition:

- The "usable spacecraft volume" remaining in the pressure vessel after accounting for the losses caused by deployed equipment, outfitting, structure, stowage, and structural inefficiencies ("nooks and crannies"). [Rudisill 08, Simon 10b]

- The space that the crew has to live and work within a spacecraft interior [Simon 10b]

- Quantification Method:

- Numerical estimation using Boolean collision tests to identify usable volume [Simon 10b]

- References:

- Requirements: Celentano 63, Fraser 68, NASA 95, Rudisill 08, NASA 10

- Quantification: Cohen 08, Szabo 07, Simon 10b
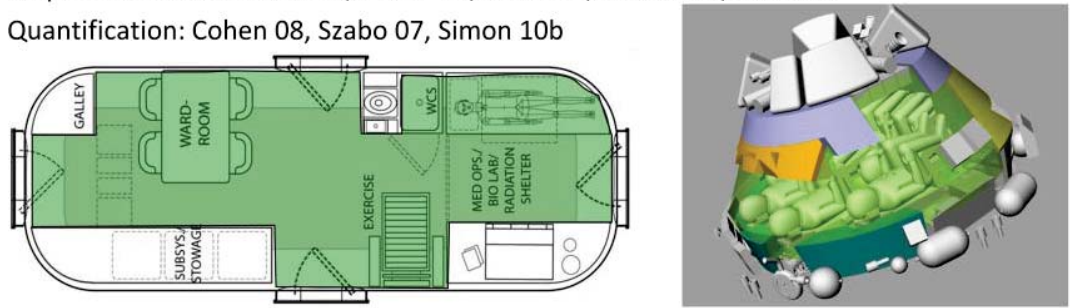

Figure 18: Example of Evaluation Criteria Information: Habitable Volume

\section{Separation for Privacy (Quantifiable using Proxy Measurements)}

- Inputs: Interior subsystem locations, associated subsystem functions, separation relationships [NASA 10 , Tullis 88]

- Definition:

- A measure of the separation of public functions from private functions ranked by degree of desired separation (ex. Sleeping quarters and wardroom) [Tullis 88]

- Quantification Method:

- Functional relationships analysis using the Euclidean-norm of the Hadamard product of separation relationships and Euclidean distance matrices [Tullis 88]

- References:

- Requirements: Tullis 88, NASA 95, NASA 10

- Quantification: Tullis 88
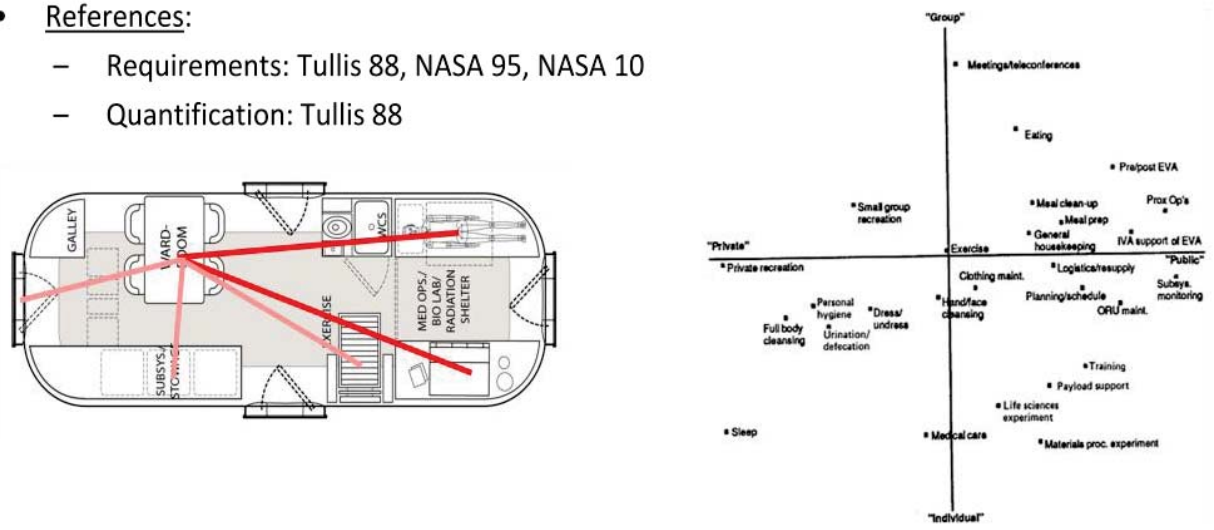

Figure 19: Example of Evaluation Criteria Information: Separation for Privacy 


\section{Line Run Mass (Quantitative Proxy)}

- Inputs: Locations of systems or equipment, specification of what kind of line runs apply to each piece of equipment

- Definition:

- The mass of power distribution, atmosphere distribution, vacuum, and various water distribution lines are highly dependent upon the placement of interior objects which connect to these lines and the amount of material to be transported within the habitat. Colocation of systems sharing a certain type of line can reduce this mass by reducing the length of the line run.

- Quantification Method:

- Determine the minimum path length connecting the objects sharing a particular type of line run through mathematical calculation of distances (Manhattan or Euclidean) between objects for a full factorial set of possible path orders (ABCD, ABDC, BDCA, etc.)

- References:

- Quantification: Anderson and Bagdigian (phone conversations)

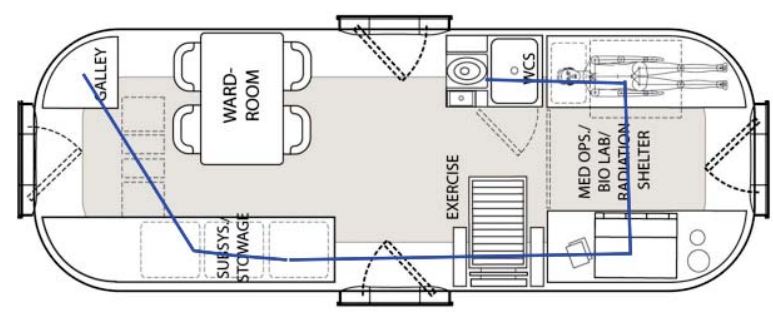

\section{Unusable Volume (Quantifiable)}

- Inputs: Pressure vessel geometry, interior subsystems geometries and locations, astronaut anthropometric geometries

- Definition:

- This is a measure of the inaccessible volume and structural inefficiencies caused by the particular packing strategy [Rudisill 08 , Simon 10b]

- Unoccupied, inaccessible volume (Space which is within the pressure shell, but not included in in habitable, hardware, or stowage volumes)

- Quantification Method:

- Numerical estimation using Boolean collision tests to identify wasted volume [Simon 10b]

- References:

- Requirements: No requirement - Quantification: Simon 10b

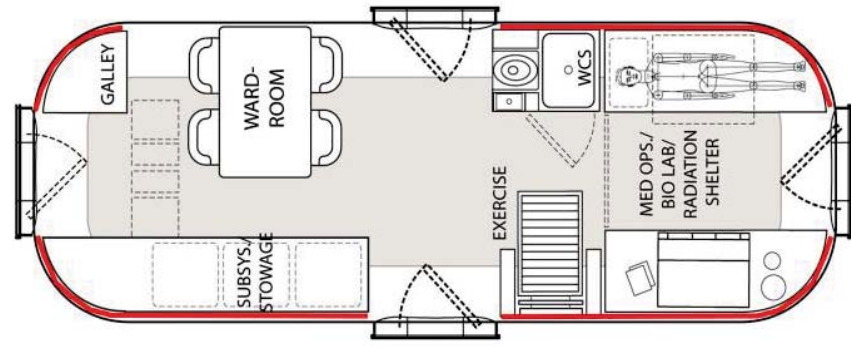




\section{Largest Spatial Vista (Quantifiable)}

- $\underline{\text { Inputs: }}$ Pressure vessel geometry, interior subsystems geometries and locations, astronaut anthropometric dimensions

- Definition:

- Spatial vista/maximum contiguous line of sight/ contiguous field of view is a measure the maximum volume swept by the eye of a crew member. This is a measure of spaciousness and psychological/physiological acceptability of the environment. This is also a measure of the quality of the interior volume [NASA 10, SICSA 08]

- Quantification Method:

- Numerical estimation using Boolean collision tests to identify "line-of-sight" lengths which can be integrated to calculate visible volume [Simon 10a]

- References:

- Requirements: No requirement

- Quantification: NASA 10, Simon 10
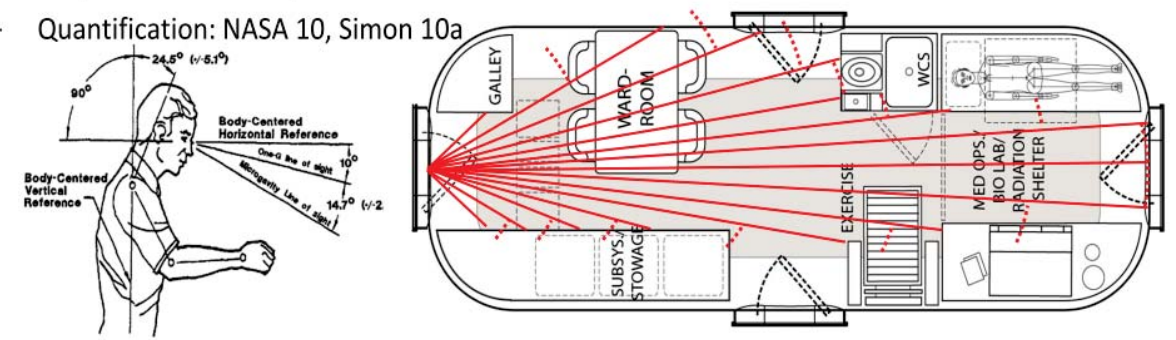

\section{Anthropometry Interferences of High Duration Tasks (Quantifiable)}

- Inputs: Pressure vessel geometry, interior subsystems geometries and locations, astronaut anthropometric geometries associated with each piece of hardware, durations of tasks

- Definition:

- This is a measure of the number of long duration tasks whose anthropometric volumes interfere with either the anthropometric volumes of other tasks, translation paths, or hatch clearance areas.[Fitts 02, NASA 10, Simon 10a]

- Quantification Method:

- Counting collisions detected between anthropometric volumes associated with each highduration function and other high-duration functions, translation paths, and hatch clearance areas [Fitts 02, Simon 10a]

- References:

- Requirements: Fitts 02, NASA 10

- Quantification: Tullis 88, Simon 10a

High-Duration Anthropometric Volumes

Translation Paths

Interferences Detected

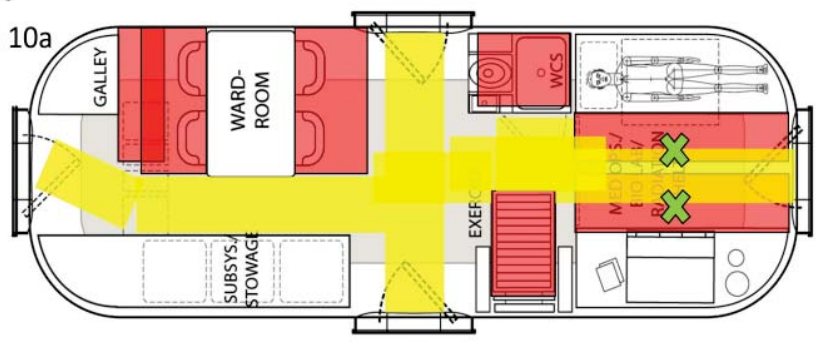




\section{Placement for High Frequency/Duration Use (Quantifiable using Proxy Measurements)}

- Inputs: Ranges of acceptable positions for use of objects, nominal value for position for frequent use of object, actual locations of various objects, frequency of use and duration of use for each object [Tullis 88, NASA 10]

- Definition:

- Rather than placing equipment based upon relationships to other interior systems, this criteria measures the appropriateness of placement based upon the need for comfortable, longduration or frequent access to a piece of equipment. (i.e. between waist and shoulder heights)

- Quantification Method:

- Calculate a weighted sum of utilities functions (Functions defined by optimal location as in ergonomics placement evaluation criteria and weightings normalized by frequency/duration of use). Sum for all objects. [Simon 10a]

- $\quad$ References

- Requirements: NASA 10

- Quantification: Tullis 88, Simon 10a

High Frequency/Duration Access

Low Frequency/Duration Access

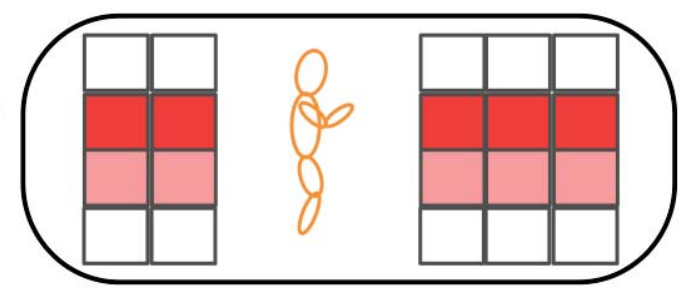

\section{Separation of Clean and Dirty Zones (Quantifiable using Proxy Measurements)}

- Inputs: Interior subsystem locations, associated subsystem functions, separation relationships [NASA 10, Tullis 88]

- Definition:

- A measure of the separation of clean function/zones (e.g. crew quarters) from dirty functions/zones (e.g. EVA workstations) ranked by degree of desired separation [Tullis 88]

- Quantification Method:

- Functional relationships analysis using the Euclidean-norm of the Hadamard product of separation relationships and Euclidean distance matrices [Tullis 88]

- References:

- Requirements: Tullis 88, NASA 95, NASA 10

- Quantification: Tullis 88

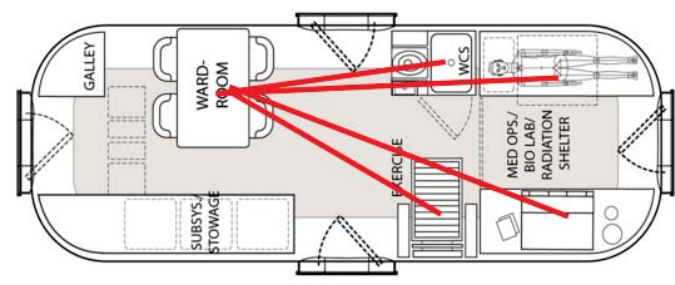




\section{Separation for Noise (Quantifiable using Proxy Measurements)}

- Inputs: Interior subsystem locations, associated subsystem functions, separation relationships [NASA 10, Tullis 88]

- Definition:

- A measure of the separation of noisy functions from functions requiring noise isolation ranked by degree of desired separation (ex. Sleeping quarters and wardroom) [Tullis 88]

- Quantification Method:

- Functional relationships analysis using the Euclidean-norm of the Hadamard product of separation relationships and Euclidean distance matrices [Tullis 88]

- References:

- Requirements: Tullis 88, NASA 95, NASA 10

- Quantification: Tullis 88

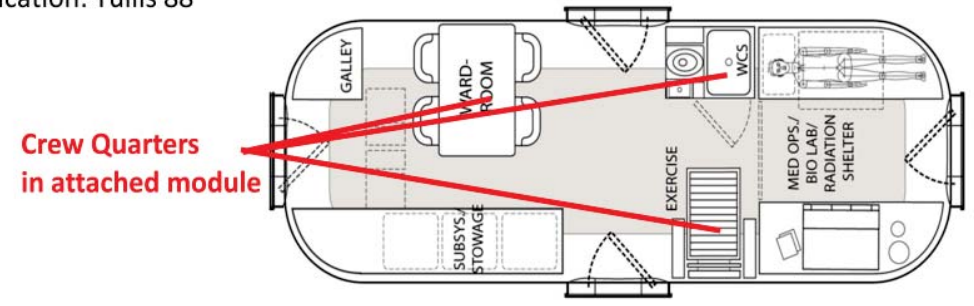

